\title{
On finding approximate optimal paths in weighted regions
}

\author{
Zheng Sun ${ }^{\mathrm{a}, *, 1}$, John H. Reif ${ }^{\mathrm{b}, 2}$ \\ a Department of Computer Science, Hong Kong Baptist University, Kowloon, Hong Kong \\ b Department of Computer Science, Duke University, Box 90129, Durham, NC 27708-0129, USA
}

Received 22 June 2003

Available online 3 September 2004

\begin{abstract}
The main result of this paper is an approximation algorithm for the weighted region optimal path problem. In this problem, a point robot moves in a planar space composed of $n$ triangular regions, each of which is associated with a positive unit weight. The objective is to find, for given source and destination points $s$ and $t$, a path from $s$ to $t$ with the minimum weighted length. Our algorithm, BUSHWHACK, adopts a traditional approach (see [M. Lanthier, A. Maheshwari, J.-R. Sack, Approximating weighted shortest paths on polyhedral surfaces, in: Proceedings of the 13th Annual ACM Symposium on Coputational Geometry, 1997, pp. 274-283; L. Aleksandrov, M. Lanthier, A. Maheshwari, J.-R. Sack, An $\varepsilon$-approximation algorithm for weighted shortest paths on polyhedral surfaces, in: Proceedings of the 6th Scandinavian Workshop on Algorithm Theory, in: Lecture Notes in Comput. Sci., vol. 1432, 1998, pp. 11-22; L. Aleksandrov, A. Maheshwari, J.-R. Sack, Approximation algorithms for geometric shortest path problems, in: Proceedings of the 32nd Annual ACM Symposium on Theory of Computing, 2000, pp. 286-295]) that converts the original continuous geometric search space into a discrete graph $\mathcal{G}$ by placing representative points on boundary edges. However, by exploiting geometric structures that we call intervals, BUSHWHACK computes
\end{abstract}

\footnotetext{
is Parts of this work were published in Proceedings of the 4th Workshop on Foundations of Algorithmic Robotics, 2000, pp. 191-203, and in Proceedings of the 14th International Symposium on Fundamentals of Computation Theory, in: Lecture Notes in Comput. Sci., vol. 2751, 2003, pp. 258-270.

* Corresponding author.

E-mail addresses: sunz@comp.hkbu.edu.hk (Z.Sun), reif@cs.duke.edu (J.H. Reif).

1 Supported in part by Hong Kong RGC Grant HKBU2107/04E.

2 Supported in part by NSF ITR Grants EIA-0086015 and 0326157, NSF QuBIC Grants EIA-0218376 and EIA-0218359, and DARPA/AFSOR Contract F30602-01-2-0561.
} 
an approximate optimal path more efficiently as it accesses only a sparse subgraph of $\mathcal{G}$. Combined with the logarithmic discretization scheme introduced by Aleksandrov et al. [Approximation algorithms for geometric shortest path problems, in: Proceedings of the 32nd Annual ACM Symposium on Theory of Computing, 2000, pp. 286-295], BUSHWHACK can compute an $\varepsilon$-approximation in $O\left(\frac{n}{\varepsilon}\left(\log \frac{1}{\varepsilon}+\log n\right) \log \frac{1}{\varepsilon}\right)$ time. By reducing complexity dependency on $\varepsilon$, this result improves on all previous results with the same discretization approach. We also provide an improvement over the discretization scheme of [L. Aleksandrov, A. Maheshwari, J.-R. Sack, Approximation algorithms for geometric shortest path problems, in: Proceedings of the 32nd Annual ACM Symposium on Theory of Computing, 2000, pp. 286-295] so that the size of $\mathcal{G}$ is no longer dependent on unit weight ratio, the ratio between the maximum and minimum unit weights. This leads to the first $\varepsilon$-approximation algorithm whose time complexity does not depend on unit weight ratio.

(c) 2004 Elsevier Inc. All rights reserved.

Keywords: Robotics; Computational geometry; Approximation algorithms; Optimal paths

\section{Introduction}

\subsection{Definition of the problem}

Optimal path planning problems are some of the most fundamental problems in robotics research. Each optimal path problem is to determine an optimal path (a shortest path according to a user-defined metric on paths) between two designated points $s$ and $t$ in a $2 \mathcal{D}$ or $3 \mathcal{D}$ space under various conditions. Due to their practical significance, these problems have drawn great attention from researchers in robotics as well as other areas such as computational geometry, geographical information systems (GIS), and graph theory. Interested readers may refer to survey [5] for a comprehensive review of the previous works on these problems.

In this paper we study the $2 \mathcal{D}$ weighted region optimal path problem. In this problem, a $2 \mathcal{D}$ space is divided into $n$ triangular regions, each of which is associated with a distinct positive unit weight. Such a space can be used to model an area consisting of different geographical features, such as deserts, forests, grasslands, and lakes, in which the traveling costs for the robot are different. The cost of a path $p$ in this space is defined to be the weighted length of $p$, and is denoted by $\|p\|$. The objective is to find an optimal path, the path with the minimum cost, from a source point $s$ to a destination point $t$. In some literature, this problem is called the "homogeneous-cost region path planning problem." Figure 1 shows an optimal path in a $2 \mathcal{D}$ space with four triangular weighted regions. In the figure, the darker a region is marked, the greater the unit weight of the region is.

Observe that the unweighted optimal path problem can be considered as a special case of the weighted optimal path problem: for any point in the "free space," the unit weight is defined to be 1; for any point in the "obstacle" (if there is any), the unit weight is defined to be $+\infty$. The key difference between $2 \mathcal{D}$ weighted and unweighted optimal path problems is that, while for the unweighted case an optimal path only bends at obstacle vertices, for the weighted case an optimal path may bend at any point on a boundary edge separating two regions with different unit weights. This problem is generally considered to be very hard 


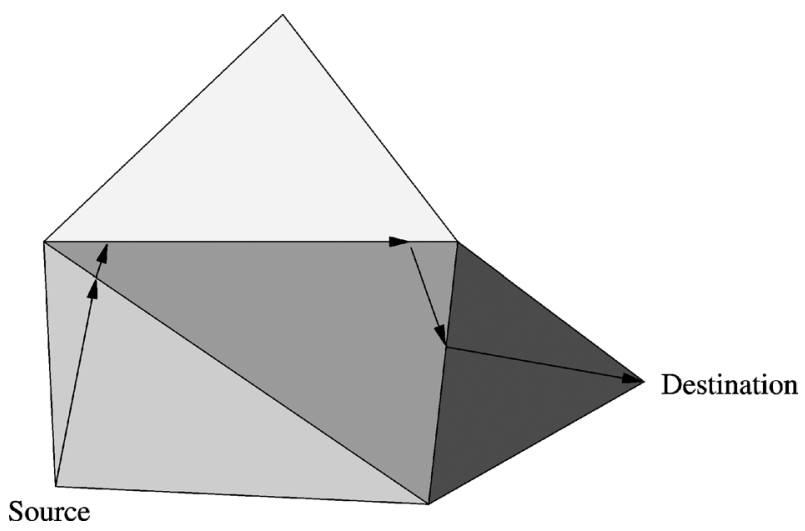

Fig. 1. Optimal path in weighted regions.

and therefore most of the known research works have focused on various approximation algorithms.

For any points $s$ and $t$, we use $p_{\text {opt }}(s, t)$ to denote an optimal path from $s$ to $t$ and use $p_{\text {opt }}^{\prime}(s, t)$ to denote an approximate optimal path from $s$ to $t$ computed by a given approximation algorithm. We decompose the error of the approximation, $\left\|p_{\mathrm{opt}}^{\prime}(s, t)\right\|-$ $\left\|p_{\text {opt }}(s, t)\right\|$, into two components: the absolute error (or additive error) and the relative error (or multiplicative error). The absolute error is bounded by a constant value disregarding the value of $\left\|p_{\text {opt }}(s, t)\right\|$, while the relative error is linear in $\left\|p_{\text {opt }}(s, t)\right\|$. We say that the error of the approximation is $\varepsilon$-relative and $\delta$-absolute if $\left\|p_{\text {opt }}^{\prime}(s, t)\right\|-\left\|p_{\text {opt }}(s, t)\right\|=$ $\varepsilon \cdot\left\|p_{\text {opt }}(s, t)\right\|+\delta$. In this paper we mainly study $\varepsilon$-approximation algorithms that guarantee to produce $\varepsilon$-good approximate optimal paths, approximate optimal paths with no absolute error but just relative error.

In the following we will discuss our work and other related work in the context of planar weighted region optimal path problem. It is important to note that most of the results on weighted region optimal path problem, including the ones we will present in the following sections, can also be applied to weighted polyhedral surfaces.

\subsection{Previous works}

We first define some parameters that will be used in the complexity analysis of various approximation algorithms. We let $n$ be the number of triangular regions in the plane, and let $L$ be the length of the longest boundary edge of all regions. For our convenience, we assume that the coordinates of the vertices are all non-negative integers, and let $N$ be the maximum coordinate. We use $w_{\max }\left(w_{\min }\right.$, respectively) to denote the maximum (minimum, respectively) unit weight among all regions, and define the unit weight ratio $\mu$ to be $w_{\max } / w_{\min }$.

One of the earliest $\varepsilon$-approximation algorithms on this problem was provided by Mitchell and Papadimitriou [6]. Their algorithm uses "Snell's Law of Refraction" and the continuous Dijkstra method to give an optimal-path map for a given source point $s$. 
Table 1

Performance parameters

\begin{tabular}{ll}
\hline$\varepsilon$ & the user-defined relative error allowed \\
$n$ & number of regions \\
$L$ & length of the longest edge \\
$N$ & maximum coordinate of the vertices \\
$w_{\max }\left(w_{\min }\right)$ & the maximum (minimum) unit weight \\
$\mu$ & the unit weight ratio \\
$\theta_{\min }$ & minimum angle between two adjacent boundary edges \\
\hline
\end{tabular}

The time complexity of their algorithm is $O\left(n^{8} \log \frac{n N \mu}{\varepsilon}\right)$. In practice, however, the time complexity is expected to be much lower.

Mata and Mitchell [7] provided an alternative algorithm using edge subdivision. This algorithm discretizes the original continuous space by placing with even spacing $m$ representative points (which we call Steiner points) along each boundary edge. Then it constructs a discrete graph $\mathcal{G}$ that includes these Steiner points as well as the vertices of the triangular subdivision. For any two discrete points (vertices or Steiner points) $v_{1}$ and $v_{2}$ in $\mathcal{G}$ that are on the border of the same region, an edge $\left(v_{1}, v_{2}\right)$ is added into $\mathcal{G}$ with an assigned weight of the weighted length of segment $\overline{v_{1} v_{2}}$. Each path in $\mathcal{G}$, which we call a discrete path, corresponds to a path with the same cost in the original space. An optimal discrete path (the minimum-cost path among all discrete paths in $\mathcal{G}$ ) from $s$ to $t$ is then computed in $O\left(n m^{2}+n m \log n m\right)$ time using Dijkstra's algorithm. Depending on the quality of the discretization, this optimal discrete path usually gives a good approximation to a real optimal path from $s$ to $t$. Lanthier et al. [1] independently provided an $O\left(n^{5}\right)$ algorithm using a uniform discretization that guarantees an absolute error of $O\left(L w_{\max }\right)$ by choosing $m=n^{2}$. This uniform discretization method was also used earlier by Smith et al. [8].

Aleksandrov et al. [2,3] later provided two logarithmic discretization methods. For a given $\varepsilon$, either of the two discretization methods constructs, by placing $m=O\left(\frac{1}{\varepsilon} \log \frac{1}{\varepsilon}\right)$ Steiner points on each boundary edge, a graph $\mathcal{G}_{\varepsilon}$ that can be guaranteed to contain an $\varepsilon$ good approximate optimal path from $s$ to $t$. Therefore, it takes $O\left(\frac{n}{\varepsilon}\left(\frac{1}{\varepsilon} \log \frac{1}{\varepsilon}+\log n\right) \log \frac{1}{\varepsilon}\right)$ time to find an $\varepsilon$-good approximate optimal path using any of the two discretization methods. Similar to the other algorithms, the complexity also involves a number of geometric parameters such as $L, \mu, \theta_{\min }$, etc.

The time complexities of the above edge-subdivision-based algorithms are rather high, as $\mathcal{G}$ is a dense graph with $O\left(\mathrm{~nm}^{2}\right)$ edges. To reduce the cost of processing the edges of $\mathcal{G}$, alternative algorithms that only access a subgraph of $\mathcal{G}$ were investigated. For example, with the uniform discretization, Lanthier et al. [1] proposed an algorithm that only computes an optimal discrete path in an $\varepsilon$-spanner of $\mathcal{G}$. The time complexity is reduced to $O\left(\frac{n^{3}}{\varepsilon}+n^{3} \log n\right)$, as each point is selectively connected to $O\left(\frac{1}{\varepsilon}\right)$ other points. In addition to the absolute error of $O\left(L w_{\max }\right)$ introduced by the discretization, the approximate optimal path found by this algorithm also has a relative error of $\varepsilon$ introduced by using the spanner.

The best result is provided by [3]. Their pruned Dijkstra's algorithm uses two stages to trim the edges of $\mathcal{G}$. First, by exploiting Snell's Law they define a geodesic cone for 
Table 2

Complexity comparison

\begin{tabular}{lll}
\hline Algorithm & Complexity & Error \\
\hline Mitchell and Papadimitriou [6] & $O\left(n^{8} \log \frac{n}{\varepsilon}\right)$ & $\varepsilon$-relative \\
Lanthier et al. [1] & $O\left(n^{5}\right)$ & $O\left(L w_{\text {max }}\right)$-absolute \\
Lanthier et al. [1] & $O\left(\frac{n^{3}}{\varepsilon}+n^{3} \log n\right)$ & $\varepsilon$-relative and \\
Aleksandrov et al. [2] & $O\left(\frac{n}{\varepsilon}\left(\frac{1}{\varepsilon}+\log n\right) \log \frac{1}{\varepsilon}\right)$ & $O\left(L w_{\max }\right)$-absolute \\
Aleksandrov et al. [3] & $O\left(\frac{n}{\varepsilon}\left(\frac{1}{\sqrt{\varepsilon}}+\log n\right) \log \frac{1}{\varepsilon}\right)$ & $\varepsilon$-relative \\
This work & $O\left(\frac{n}{\varepsilon}\left(\log \frac{1}{\varepsilon}+\log n\right) \log \frac{1}{\varepsilon}\right)$ & $\varepsilon$-relative \\
\hline
\end{tabular}

each Steiner point $v$, claiming that any optimal discrete path passing through $v$ can only leave $v$ via an edge inside this cone. The second stage is to construct an $\varepsilon$-spanner for the geodesic cone of each Steiner point. The number of incident edges of each Steiner point is therefore reduced to $O\left(\frac{1}{\sqrt{\varepsilon}}\right)$, resulting in an $O\left(\frac{n}{\varepsilon}\left(\frac{1}{\sqrt{\varepsilon}}+\log n\right) \log \frac{1}{\varepsilon}\right)$ algorithm. Although the $\varepsilon$-spanner causes an additional term of error, the approximate optimal path computed remains $O(\varepsilon)$-good.

In Table 2, we list the time complexity and error bound of each of the above-mentioned algorithms for a comparison. For our convenience, we omit all geometric parameters and state the complexity of each algorithm in terms of $n$ and $\varepsilon$. It is important to note, however, that algorithms following different approaches cannot be compared using merely dependencies on $n$ and $\varepsilon$, because the running time may have significantly different dependencies on other geometric parameters. For example, the running time of Mitchell and Papadimitriou's algorithm [6] depends on $N$ only logarithmically, while that of Aleksandrov et al.'s algorithm [2] has a linear dependency on $1 / \theta_{\min }$, which, in the worst case, could be $\Omega\left(N^{2}\right)$.

For other related work, see [9-16], or see survey [5].

\section{Preliminaries}

\subsection{Notations}

Let $S$ be a planar space consisting of $n$ triangular regions. We use $V$ and $E$ to denote the set of vertices and the set of boundary edges, respectively, of all regions. Therefore, $|V|=O(n)$, and $|E|=O(n)$. For any region $r$, we use $w_{r}$ to denote the unit weight of $r$. For each boundary edge $e \in E$, the unit weight $w_{e}$ is defined to be $\min \left\{w_{r}, w_{r^{\prime}}\right\}$, where $r$ and $r^{\prime}$ are the two regions incident to $e$. For any points $v_{1}$ and $v_{2}$ in a region $r$, we define $d_{r}\left(v_{1}, v_{2}\right)=w_{r} \cdot\left|\overline{v_{1} v_{2}}\right|$ to be the distance in the region between $v_{1}$ and $v_{2}$. Similarly, we say that $d_{e}\left(v_{1}, v_{2}\right)=w_{e} \cdot\left|\overline{v_{1} v_{2}}\right|$ is the distance along the edge between $v_{1}$ and $v_{2}$ for any points $v_{1}$ and $v_{2}$ on boundary edge $e$. Let $s$ and $t$ be the designated source point and destination point, respectively. We assume that $s, t \in V$. If otherwise, we can add a constant number of boundary edges to construct from $S$ a new triangular decomposition $S^{\prime}$ that has $s$ and $t$ as vertices. 
It has been proved (see [6]) that a weighted region optimal path is a piecewise linear path consisting of $O\left(n^{2}\right)$ segments. The two endpoints of each segment are on the boundary of the same region. A segment of a path is said to be "edge-crawling" if it lies on a boundary edge. Similarly, a segment is "face-crossing" if it cuts through a region. We call a path a "face-crossing" ("edge-crawling") path if the last segment of the path is "face-crossing" ("edge-crawling," respectively).

For any path $p$, we use $|p|$ and $\|p\|$ to denote its Euclidean length and weighted length, respectively. For any two points $v_{1}, v_{2}$ on a path $p$, let $p\left[v_{1}, v_{2}\right]$ denote the part of $p$ between $v_{1}$ and $v_{2}$. For two paths $p_{1}$ and $p_{2}$ sharing one endpoint, we let $p_{1}+p_{2}$ denote the concatenation of $p_{1}$ and $p_{2}$. If $p=p_{1}+p_{2}$, we say that $p$ is an extension of $p_{1}$. In particular, if $p=p_{1}+\overline{v_{1} v_{2}}$, where $v_{1}$ is one of the endpoints of $p_{1}$, we call $p$ a onesegment extension of $p_{1}$. For any two points $x$ and $y$, we use $p(x, y)$ to denote a path from $x$ to $y$ and use $p_{\text {opt }}(x, y)$ to denote an optimal path from $x$ to $y$. We define the "distance" from $s$ to $t, d_{\text {opt }}(s, t)$, to be the cost of $p_{\text {opt }}(s, t)$.

Following the same approach used by [1-3], we discretize the $2 \mathcal{D}$ space by introducing Steiner points on boundary edges. For each boundary edge $e \in E$, we add $m$ Steiner points on $e$ for some positive integer $m$. Let $V_{s}$ be the set of Steiner points and let $V^{\prime}=V \cup V_{s}$. A weighted graph $\mathcal{G}\left(V^{\prime}, E^{\prime}\right)$ is constructed by interconnecting points in $V^{\prime}$ that are on the boundary of the same region. Each edge $(x, y)$ in $\mathcal{G}$ is assigned a weight $\omega(x, y)$, which is defined to be $d_{e}(x, y)$ if $\overline{x y}$ is an edge-crawling segment on boundary edge $e$; or $d_{r}(x, y)$ if $\overline{x y}$ is a face-crossing segment in region $r$.

By constructing $\mathcal{G}$, the original path planning problem in a continuous space is transformed to the problem of finding an optimal discrete path connecting $s$ and $t$ in $\mathcal{G}$. This optimal discrete path is then used to approximate a real optimal path in the original continuous space. As the goal of our algorithm is to find an optimal discrete path, in the following discussion, wherever we say a discrete path is "optimal," we mean that it is optimal among all discrete paths. The phrases "optimal discrete path" and "approximate optimal path" are used interchangeably, and are both denoted by $p_{\text {opt }}^{\prime}(s, t)$. Let $d_{\text {opt }}^{\prime}(s, t)$ be the cost of $p_{\text {opt }}^{\prime}(s, t)$. At any time during the search of an optimal discrete path from $s$ to $t$, a point $v$ is said to be discovered if and only if $d_{\text {opt }}^{\prime}(s, v)$ is determined. For each boundary edge $e$ we use $\mathrm{PLIST}_{e}$ to denote the list that includes all discovered points on $e$.

\subsection{Our approach}

\subsubsection{BUSHWHACK algorithm}

Instead of applying Dijkstra's algorithm to the resulting graph $\mathcal{G}$, our approximation algorithm uses a new discrete search algorithm called BUSHWHACK. The BUSHWHACK algorithm can compute optimal discrete paths on both uniform and logarithmic discretizations more efficiently than the ones proposed in [1] and [2,3]. For each Steiner point $v$, BUSHWHACK dynamically maintains a small set of incident edges of $v$ that may contribute to an optimal discrete path from $s$ to $t$. If $m$ Steiner points are placed on each boundary edge, during the entire computation the number of edges accessed in each region is $O(m \log m)$, and therefore the complexity of the algorithm is reduced to $O(\mathrm{~nm} \log \mathrm{nm})$, as compared to $O\left(n m^{2}+n m \log n m\right)$ of Dijkstra's algorithm. 


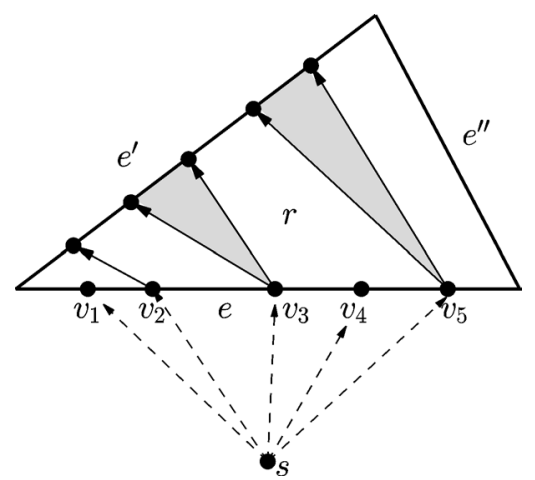

(a) On average each interval contains $O(1)$ Steiner points.

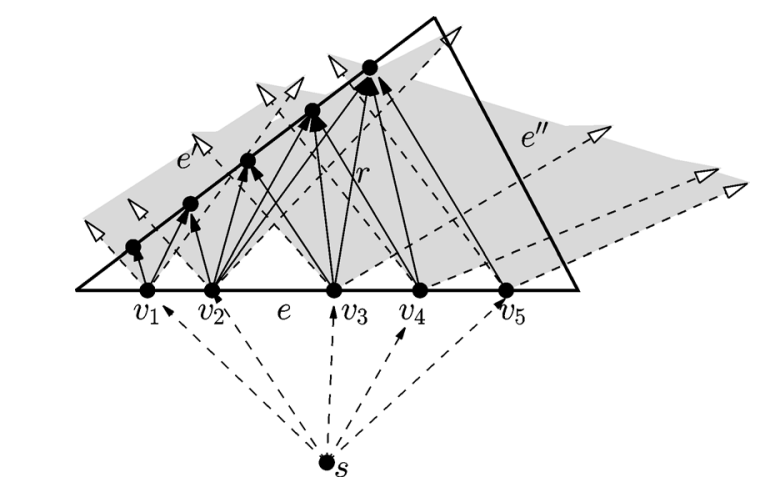

(b) Geodesic cones may overlap and each contains $O\left(\frac{1}{\sqrt{\varepsilon}}\right)$ Steiner points.

Fig. 2. Comparison between interval and geodesic cone.

Let $r$ be a weighted region and let $e, e^{\prime}$ be two boundary edges of $r$. For each point $v \in e$, we define an interval $I_{v, e, e^{\prime}}$ to be a set of contiguous Steiner points on $e^{\prime}$ such that, for any point $v^{\prime} \in e^{\prime}$, edge $\overline{v v^{\prime}}$ may be "used" by an optimal discrete path originating from $s$ only if $v^{\prime}$ is inside the interval. Each point $v^{\prime} \in I_{v, e, e^{\prime}}$ is associated with an almost-optimal path from $s$ to $v^{\prime}$ that passes through $v$. This path is a concatenation of an optimal discrete path from $s$ to $v$ and segment $\overline{v v^{\prime}}$. Moreover, this path is the least costly path among all paths from $s$ to $v^{\prime}$ that enter region $r$ through Steiner points on $e$.

The concept of intervals is similar to the geodesic cones used by Aleksandrov et al. [3]. The main difference is that, for any two points $v_{1}$ and $v_{2}$ on edge $e$, the intervals associated with $v_{1}$ and $v_{2}$ are mutually exclusive, as it will be clear later when we give the formal definition for intervals. Therefore, on average the number of Steiner points in each interval is $O(1)$, assuming that $e$ and $e^{\prime}$ contain roughly the same number of Steiner points. The geodesic cones, however, may overlap with each other as shown in Fig. 2(b). In fact, using Aleksandrov et al.'s discretization scheme [3], the number of Steiner points of each geodesic cone can only be bounded by $O\left(\frac{1}{\sqrt{\varepsilon}}\right)$. (This is still the case even if an $\varepsilon$-spanner of each geodesic cone is used.) 
Our definition of intervals is actually more related to that of the intervals of optimality used by Mitchell and Papadimitriou [6]. For the clarity of the following discussion, we call their intervals of optimality continuous intervals and our intervals discrete intervals. Each interval (continuous or discrete) consists of points to which the optimal paths have the same discrete structure. Each continuous interval describes a segment of a boundary edge so that the optimal paths to the points in the segment go through the same sequence of boundary edges. Each discrete interval describes the set of discrete points on a boundary edge so that the almost-optimal paths to these points go through the same sequence of bending points. The difference is that, while continuous intervals are treated in the continuum, discrete intervals are sets of discrete points.

Because of the difference in the definitions, our data structures and procedures for processing the discrete intervals are totally different from those found in Mitchell and Papadimitriou's algorithm, although in some cases we follow the terminologies used in [6]. Because almost-optimal paths to the points in each discrete interval go through the same sequence of bending Steiner points (not just boundary edges), we do not need to compute reverse ray tracing, which is done by the Find-Point procedure in Mitchell and Papadimitriou's algorithm. Reverse ray-tracing is a very costly operation that takes $O\left(n^{4} \log \frac{1}{\varepsilon}\right)$ time.

The number of continuous intervals is $\Omega\left(n^{4}\right)$ in the worst case, while the number of discrete intervals is proportional to the number of Steiner points, which is $O\left(\frac{n}{\varepsilon} \log \frac{1}{\varepsilon}\right)$ if the logarithmic discretization of [3] is used. As a result, the complexity of our algorithm is more dependent on $\varepsilon$, while that of Mitchell and Papadimitriou's algorithm is more dependent on $n$. It is worth mentioning that, although in the worst case the dependency of Mitchell and Papadimitriou's algorithm on $n$ is very high $\left(O\left(n^{8}\right)\right)$, their algorithm appears to be the only one that has logarithmic dependency on $\varepsilon$.

One key difference between our algorithm and the approximation algorithms of [3] and [6] is that, while these previous algorithms exploit the fact that an optimal path obeys Snell's Law when crossing boundary edges, our algorithm only uses the simple property that two optimal paths originating from the same source point cannot intersect in the interior of any region. Therefore, our algorithm is more flexible, allowing for many other heuristic cost criteria (e.g., charging a varying cost on crossing boundary edges) to be added easily.

Maintaining and updating interval information for each point $v$ involves an extra cost of accessing $O(\log m)$ incident edges of $v$. Therefore, on average, our algorithm uses $O(\log m)$ edges for each point in $\mathcal{G}$. When the uniform discretization of [1] is used, our algorithm improves the time complexity to $O\left(n^{3} \log n\right)$ (as opposed to $O\left(\frac{n^{3}}{\varepsilon}+n^{3} \log n\right)$ of [1]). With the logarithmic discretization of [3], our algorithm can find an $\varepsilon$-good approximate optimal path in $O\left(\frac{n}{\varepsilon}\left(\log \frac{1}{\varepsilon}+\log n\right) \log \frac{1}{\varepsilon}\right)$ time. This result improves on the $O\left(\frac{n}{\varepsilon}\left(\frac{1}{\varepsilon}+\log n\right) \log \frac{1}{\varepsilon}\right)$ algorithm of [2] or the $O\left(\frac{n}{\varepsilon}\left(\frac{1}{\sqrt{\varepsilon}}+\log n\right) \log \frac{1}{\varepsilon}\right)$ algorithm of [3] by a factor of $O\left(\frac{1}{\sqrt{\varepsilon}} / \log \frac{1}{\varepsilon}\right)$. Our algorithm, however, does not improve the complexity with respect to $n$.

Following our initial work of [4,17], Aleksandrov et al. [18] improves the above time complexity by another $O\left(\frac{1}{\sqrt{\varepsilon}}\right)$. They achieve so by using a novel discretization method that places Steiner points on bisectors of triangular regions instead of on boundary edges. This 
discretization method is able to reduce the number of Steiner points by a factor of $O\left(\frac{1}{\sqrt{\varepsilon}}\right)$ while still guaranteeing the same $\varepsilon$ error bound. They then apply a discrete algorithm similar to BUSHWHACK to compute an optimal discrete path in such a discretization.

\subsubsection{Compact discretization scheme}

Table 2 lists for each algorithm the time complexity with respect to $n$ and $\varepsilon$. To our best knowledge, the time complexities of all previous $\varepsilon$-approximation algorithms also depend on the unit weight ratio $\mu$, either linearly or logarithmically. This dependency is caused by the corresponding discretization scheme used. In particular, the discretization scheme of Aleksandrov et al. [3] constructs for a given $\varepsilon$ a graph $\mathcal{G}_{\varepsilon}$ with $O\left(\frac{1}{\varepsilon} \log \frac{1}{\varepsilon} \log \mu\right)$ Steiner points placed on each boundary edge. Here again we omit the other geometric parameters.

The main obstacle for removing the dependency on $\mu$ from the size of $\mathcal{G}_{\varepsilon}$ is that otherwise it is difficult to prove that for each optimal path $p_{\text {opt }}$ there exists in $\mathcal{G}_{\varepsilon}$ a discrete path that is an $\varepsilon$-approximation of $p_{\text {opt }}$. One traditional proof technique used in proving the existence of such a discrete path is to decompose $p_{\text {opt }}$ into $k$ subpaths $p_{1}, p_{2}, \ldots, p_{k}$ and then construct a discrete path $p^{\prime}=p_{1}^{\prime}+p_{2}^{\prime}+\cdots+p_{k}^{\prime}$ such that $\left\|p_{i}^{\prime}\right\| \leqslant(1+\varepsilon)\left\|p_{i}\right\|$ for each $i$. Ideally, we could choose each $p_{i}^{\prime}$ such that $p_{i}$ and $p_{i}^{\prime}$ lie in the same region, and therefore the discretization just needs to make sure that $\left|p_{i}^{\prime}\right| \leqslant(1+\varepsilon)\left|p_{i}\right|$.

However, due to the discrete nature of $\mathcal{G}_{\varepsilon}$, it is not always possible to find such $p_{i}^{\prime}$ for each $p_{i}$. For example, as shown in Fig. $3, p_{\text {opt }}$ could cross a series of boundary edges near a vertex $v$. The point where it crosses each boundary edge $e$ is between $v$ and the closest Steiner point from $v$ on $e$. To approximate $p_{\text {opt }}$ by a discrete path, one way is to replace $p_{\text {opt }}\left[u_{1}, u_{2}\right]$, the subpath of $p_{\text {opt }}$ between $u_{1}$ and $u_{2}$, by the dotted path from $u_{1}$ to $u_{2}$. However, each $p_{i}^{\prime}$ could be much longer than the corresponding $p_{i}$, thus making it impossible to bound $\left\|p_{\text {opt }}^{\prime}\right\|$ the way we described above. Instead, in the error bound proof of Aleksandrov et al. [2,3] subpath $p_{\text {opt }}\left[u_{1}, u_{2}\right]$ is replaced by the dashed path that goes through $v$. In that case, $p_{i}^{\prime}$ could travel in regions different from those in which $p_{i}$ lies in, and therefore to bound $\left\|p_{i}^{\prime}\right\|$ with respect to $\left\|p_{i}\right\|$, the discretization scheme has to take into consideration variance of unit weights.

By modifying the above proof technique, we provide in Section 6 an improvement on the discretization scheme of Aleksandrov et al. [3]. The number of Steiner points inserted

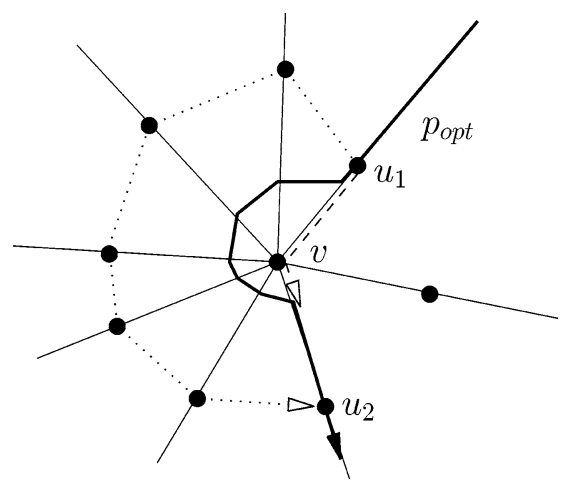

Fig. 3. "Bad" path. 
by this new discretization scheme is $O\left(\frac{1}{\varepsilon} \log \frac{1}{\varepsilon}\right)$, with the dependency on other geometric parameters unchanged. Combining BUSHWHACK with this discretization scheme, we can have the first $\varepsilon$-approximation algorithm whose time complexity is not dependent on $\mu$.

\section{Intervals}

\subsection{Disadvantage of Dijkstra's algorithm}

The BUSHWHACK algorithm works similarly to Dijkstra's algorithm. It keeps a sorted list QLIST of candidate optimal paths. At each step, the candidate optimal path $p_{\text {min }}$ with the minimum cost is extracted from QLIST. Consequently, a number of candidate optimal paths, which are one-segment extensions of $p_{\min }$, are inserted into QLIST. We call this process "path propagation." The iteration continues until the destination point is reached.

Dijkstra's algorithm can be used to compute an optimal discrete path in an arbitrary weighted graph, while the aforementioned graph $\mathcal{G}$ is derived from a $2 \mathcal{D}$ space consisting of weighted regions. Therefore, directly applying Dijkstra's algorithm to $\mathcal{G}$ does not fully utilize the underlying geometric properties.

For example, in Fig. 4 there are two paths originating from the same source point $s$. Dijkstra's algorithm will consider both paths as potential optimal discrete paths for their respective destination points. However, it is not possible that two optimal discrete paths intersect inside a weighted region, as stated by the following lemma.

Lemma 1. In a $2 \mathcal{D}$ space consisting of weighted regions, any two optimal discrete paths with the same source point cannot intersect in the interior of any region.

Proof. Assume that two optimal discrete paths $p_{\mathrm{opt}}^{\prime}\left(s, v_{1}\right)$ and $p_{\mathrm{opt}}^{\prime}\left(s, v_{2}\right)$ intersect at $b$ inside a triangular region $r$, as shown in Fig. 4. For each $i=1,2$, let $\overline{v_{i, 1} v_{i, 2}}$ be the segment

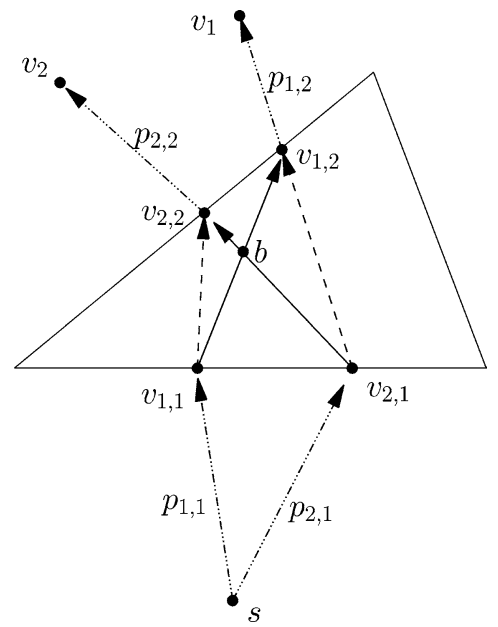

Fig. 4. Optimal discrete paths cannot intersect inside region. 
of $p_{\text {opt }}^{\prime}\left(s, v_{i}\right)$ that contains $b$. Thus, each $v_{i, j}$ is a point on the boundary of $r$. Let $p_{i, 1}=$ $p_{\text {opt }}^{\prime}\left(s, v_{i}\right)\left[s, v_{i, 1}\right]$ and let $p_{i, 2}=p_{\text {opt }}^{\prime}\left(s, v_{i}\right)\left[v_{1,2}, v_{i}\right]$. Therefore, $p_{2,1}+\overline{v_{2,1} v_{1,2}}+p_{1,2}$ is a path from $s$ to $v_{1}$ and $p_{1,1}+\overline{v_{1,1} v_{2,2}}+p_{2,2}$ is a path from $s$ to $v_{2}$. However, the total cost of the above two paths is less than that of $p_{\text {opt }}^{\prime}\left(s, v_{1}\right)$ and $p_{\text {opt }}^{\prime}\left(s, v_{2}\right)$, as $d_{r}\left(v_{1,1}, v_{1,2}\right)+$ $d_{r}\left(v_{2,1}, v_{2,2}\right)>d_{r}\left(v_{1,1}, v_{2,2}\right)+d_{r}\left(v_{2,1}, v_{1,2}\right)$. Therefore, one of $p_{\text {opt }}^{\prime}\left(s, v_{1}\right)$ and $p_{\text {opt }}^{\prime}\left(s, v_{2}\right)$ must not be optimal. This is a contradiction.

The fact that line segments $\overline{v_{1,1} v_{1,2}}$ and $\overline{v_{2,1} v_{2,2}}$ intersect inside region $r$ indicates that these two line segments cannot both contribute to optimal discrete paths originating from $s$. If we can identify useful line segments (i.e., those that may contribute to optimal discrete paths) from useless line segments, we will be able to avoid an explicit construction of the entire graph $\mathcal{G}$.

\subsection{Intervals}

To keep track of useful line segments, we introduce a data structure that we call an interval.

Definition 1. Let $r$ be a region of $S$ and let $e, e^{\prime}$ be two boundary edges of $r$. For any discovered point $v \in e$ that is not incident to $e^{\prime}$, the interval $I_{v, e, e^{\prime}}$ is defined to be

$$
\begin{gathered}
I_{v, e, e^{\prime}}=\left\{\text { discrete point } v^{*} \in e^{\prime} \mid d_{\mathrm{opt}}^{\prime}(s, v)+d_{r}\left(v, v^{*}\right) \leqslant d_{\mathrm{opt}}^{\prime}\left(s, v^{\prime}\right)+d_{r}\left(v^{\prime}, v^{*}\right)\right. \\
\left.\forall v^{\prime} \in \operatorname{PLIST}_{e}\right\} .
\end{gathered}
$$

That is, for any discrete point $v^{*}$ on $e^{\prime}, v^{*} \in I_{v, e, e^{\prime}}$ if and only if path $p_{\mathrm{opt}}^{\prime}(s, v)+\overline{v v^{*}}$ is the least costly path among all paths from $s$ to $v^{*}$ that are one-segment extensions of optimal discrete paths originating from $s$ to discovered points on $e$. In case a point $v^{*}$ on $e^{\prime}$ is included in two intervals according to this definition, we will arbitrarily assign $v^{*}$ to only one of these intervals. This is to keep intervals $I_{v, e, e^{\prime}}$ for all $v \in \operatorname{PLIST}_{e}$ mutually exclusive subsets of discrete points on $e^{\prime}$. The way the set of discrete points on a boundary edge is partitioned into intervals (or a boundary edge is partitioned into intervals of optimality as in [6]) is analogous to the way a space is partitioned into regions in a Voronoi diagram.

It is important to note that each interval $I_{v, e, e^{\prime}}$ is a dynamic set of discrete points on $e^{\prime}$. It is first created when $v$ is discovered. When more points on $e$ are discovered, PLIST $_{e}$ will contain more points and thus $I_{v, e, e^{\prime}}$ may also be changed, according to the definition.

For any edges $e$ and $e^{\prime}$ that share a region $r$, we use $\operatorname{ILIST}_{e, e^{\prime}}$ to denote the list of intervals $I_{v, e, e^{\prime}}$ for all $v \in$ PLIST $_{e}$, sorted by the Euclidean distance between $v$ and the shared endpoint of $e$ and $e^{\prime}$. Lemma 1 implies that each interval is composed of consecutive points on $e^{\prime}$ (which leads us to name this data structure "interval"). Further, an interval $I_{v, e, e^{\prime}}$ is located to the left (right, respectively) of another interval $I_{v^{\prime}, e, e^{\prime}}$ on $e^{\prime}$ if and only if $v$ is located to the left (right, respectively) of $v^{\prime}$ on $e$. Therefore, the order of discovered points on $e$ is exactly the same as the order of their corresponding intervals in $\operatorname{ILIST}_{e, e^{\prime}}$, as shown in Fig. 5.

When a Steiner point $v$ on boundary edge $e$ is newly discovered, for each boundary edge $e^{\prime}$ that shares a region $r$ with $e$, an interval $I_{v, e, e^{\prime}}$ needs to be created and inserted into 


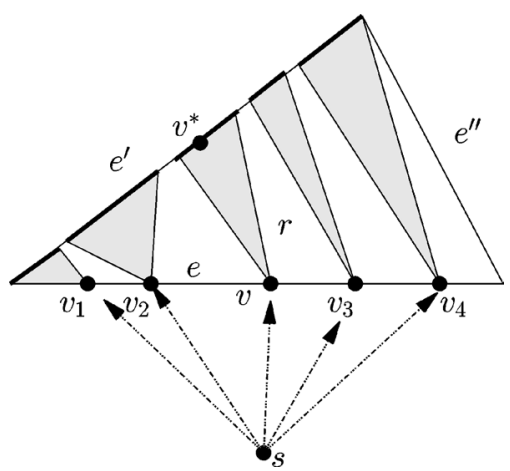

Fig. 5. Intervals.

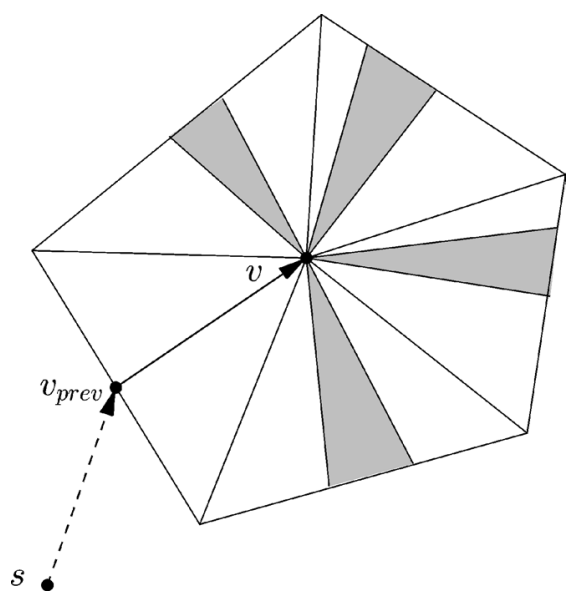

Fig. 6. Creating intervals for a vertex.
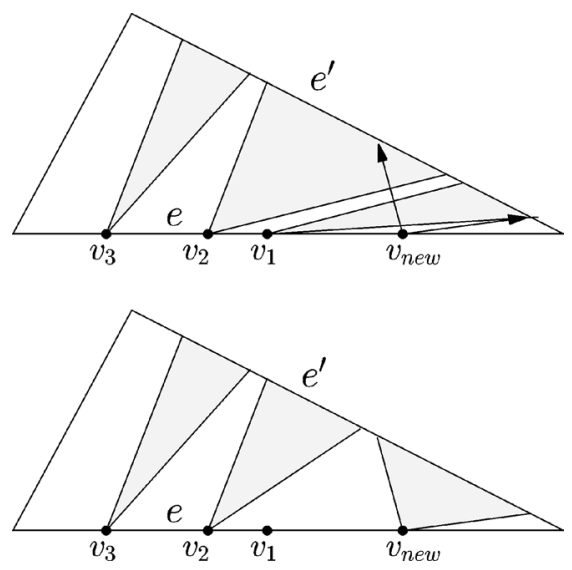

Fig. 7. Inserting an interval. 
$\operatorname{ILIST}_{e, e^{\prime}}$. In case the newly discovered point $v$ is a vertex of a triangular region, an interval needs to be created for every boundary edge facing $v$, as shown in Fig. 6 . The range of the new interval $I_{v, e, e^{\prime}}$, i.e., the two endpoints of the interval, can be computed efficiently in $O(\log m)$ time.

If the new interval $I_{v, e, e^{\prime}}$ is not empty, the ranges of the neighboring intervals need to be readjusted. Figure 7 illustrates how a new interval could totally deplete or partially deplete existing intervals. As shown in the figure, the left and right endpoints are determined for the new interval associated with $v_{\text {new }}$, the most recently discovered point. The new interval covers entirely $I_{v_{1}, e, e^{\prime}}$, the interval associated with $v_{1}$, and thus $I_{v_{1}, e, e^{\prime}}$ is totally depleted by the new interval. The interval associated with $v_{2}$ is partially depleted by the new interval as it loses part of Steiner points in it. Totally depleted intervals will be removed from $\operatorname{ILIST}_{e, e^{\prime}}$, while the range of each partially depleted interval will be readjusted.

From Definition 1 we can conclude that, for any discovered point $v$ on $e$ and any point $v^{*}$ on $e^{\prime}, \overline{v v^{*}}$ cannot be part of any optimal discrete path originating from $s$ that enters region $r$ through point $v$ if $v^{*} \notin I_{v, e, e^{\prime}}$. Therefore, by maintaining $\operatorname{ILIST}_{e, e^{\prime}}$ for each $e$ and $e^{\prime}$ that share a region, the BUSHWHACK algorithm is able to avoid accessing most of the useless edges in $\mathcal{G}$.

\subsection{Monotonic intervals}

Instead of maintaining for each $e$ and $e^{\prime}$ a list $\operatorname{ILIST}_{e, e^{\prime}}$ of intervals as defined in Definition 1, our implementation of the BUSHWHACK algorithm actually maintains a list of monotonic intervals. We first give the definition below:

Definition 2. A monotonic interval $I_{v, e, e^{\prime}}$ is an interval such that the distance (in the region) from $v$ to points in $I_{v, e, e^{\prime}}$ is monotonically increasing or decreasing along $e^{\prime}$.

Since each interval $I_{v, e, e^{\prime}}$ can only be divided to no more than two monotonic intervals (by the perpendicular point of $v$ on $e^{\prime}$ ), the total number of monotonic intervals inside each region is still linear to the number of Steiner points. In the following we will explain why we prefer to keep track of the monotonic intervals instead of the (original) intervals.

For each $v^{*} \in I_{v, e, e^{\prime}}$, line segment $\overline{v v^{*}}$ may be a useful segment and therefore we need to check the optimality of the face-crossing path $p_{\text {opt }}^{\prime}(s, v)+\overline{v v^{*}}$. We call such a path a direct interval path associated with $I_{v, e, e^{\prime}}$. One strategy is to insert all these paths into QLIST simultaneously when $v$ is discovered (and $I_{v, e, e^{\prime}}$ is created). However, this may not be efficient as both $\operatorname{ILIST}_{e, e^{\prime}}$ and intervals in $\operatorname{ILIST}_{e, e^{\prime}}$ are dynamic data structures. Whenever a new point on $e$ is discovered, a new interval (although possibly empty) will be created and inserted into $\operatorname{ILIST}_{e, e^{\prime}}$. If the new interval is not empty, the ranges of the two neighboring intervals will be adjusted.

Therefore, even though a point $v^{*}$ originally is in $I_{v, e, e^{\prime}}$, after a new point $v_{\text {new }} \in e$ is discovered, $v^{*}$ may fall into the range of the new interval $I_{v_{\text {new }}, e, e^{\prime}}$. If this is the case, path $p_{\text {opt }}^{\prime}(s, v)+\overline{v v^{*}}$ no longer needs to be considered as an optimal discrete path from $s$ to $v^{*}$ as it is more costly than $p_{\text {opt }}^{\prime}\left(s, v_{\text {new }}\right)+\overline{v_{\text {new }} v^{*}}$, according to Definition 1 .

A more efficient strategy is to insert direct interval paths in the following lazy and bestfirst manner: interval paths associated with $I_{v, e, e^{\prime}}$ are sorted in the increasing order of path 


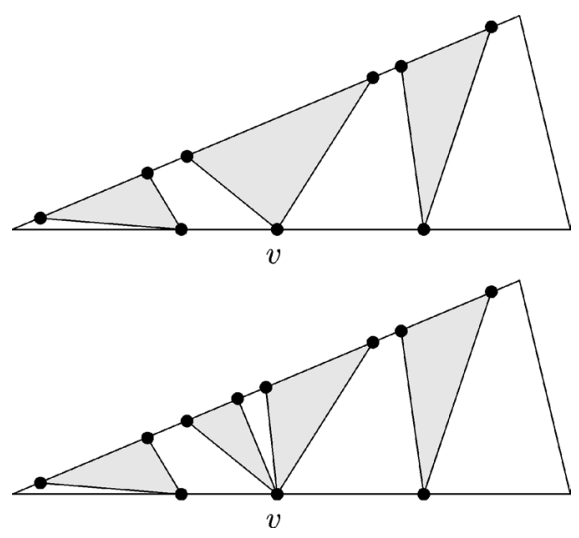

Fig. 8. Splitting an interval.

cost, and a path $p_{\text {opt }}^{\prime}(s, v)+\overline{v v^{*}}$ is inserted into QLIST only when the previous path is extracted from the list, and only if $v^{*}$ is still in $I_{v, e, e^{\prime}}$. This strategy will avoid inserting a path $p_{\text {opt }}^{\prime}(s, v)+\overline{v v^{*}}$ into QLIST if $v^{*}$ is later "switched" to another interval.

To achieve this, we need to sort efficiently the direct interval paths by path cost. Since these paths are all one-segment extensions of $p_{\text {opt }}^{\prime}(s, v)$, we only need to sort $d_{r}\left(v, v^{*}\right)$ for all $v^{*} \in I_{v, e, e^{\prime}}$. As each weighted region is a Euclidean space, each interval $I_{v, e, e^{\prime}}$ can be split into at most two parts by the perpendicular point of $v$ on $e^{\prime}$, so that the distance (in the region) from $v$ to points in each part is monotonically increasing or decreasing.

One way to get a sorted list of direct interval paths associated with $I_{v, e, e^{\prime}}$ is to do a merge-sort using these two parts. A simpler and more efficient way is to create a monotonic interval for each part of $I_{v, e, e^{\prime}}$, as illustrated in Fig. 8. The original $I_{v, e, e^{\prime}}$ is thus replaced by these intervals in $\operatorname{ILIST}_{e, e^{\prime}}$. Points in each such interval are readily sorted by distance (in the region) to $v$. Although as a result of splitting the number of intervals in $\operatorname{ILIST}_{e, e^{\prime}}$ is increased, each interval is easier to manipulate. The implementation of our algorithm follows this scheme. In the following discussion, we always assume that each interval is monotonic, and points in each interval $I_{v, e, e^{\prime}}$ are sorted by distance (in the region) to $v$.

\subsection{Propagating interval paths}

This subsection explains how the BUSHWHACK algorithm inserts into (and subsequently removes from) QLIST paths associated with each interval $I_{v, e, e^{\prime}}$. These paths include direct interval paths, as defined in the last subsection, and extended interval paths, each of which extends a direct interval path by one segment along $e^{\prime}$. Since the cost of traveling along $e^{\prime}$ may be lower than that of traveling inside the region, such an extended interval path to a point $v^{*} \in I_{v, e, e^{\prime}}$ may be less costly than a direct interval path to $v^{*}$, although its Euclidean length is larger. We call both direct interval paths and extended interval paths interval paths. By "propagating," we mean that intervals paths associated with $I_{v, e, e^{\prime}}$ will only be inserted into QLIST one at a time, and only after the previous interval path associated with the same interval is removed from QLIST. 


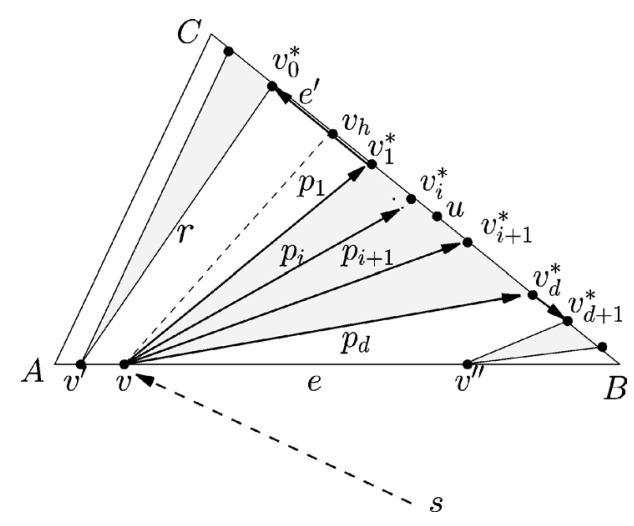

Fig. 9. Propagating interval paths.

\subsubsection{Interval paths and locally interval paths}

Following the notations used previously, we let $e$ and $e^{\prime}$ be two boundary edges of region $r$. Suppose interval $I_{v, e, e^{\prime}}$ contains points $v_{1}^{*}, v_{2}^{*}, \ldots, v_{d}^{*}$ when it is initially created (i.e., when $v$ is discovered), as shown in Fig. 9. Here $v_{1}^{*}, v_{2}^{*}, \ldots, v_{d}^{*}$ are consecutive points on $e^{\prime}$ and $v_{1}^{*}$ and $v_{d}^{*}$ are the two endpoints of the interval. Without loss of generality, we assume $d_{r}\left(v, v_{1}^{*}\right) \leqslant d_{r}\left(v, v_{d}^{*}\right)$. As interval $I_{v, e, e^{\prime}}$ is monotonic, we have $d_{r}\left(v, v_{1}^{*}\right)<d_{r}\left(v, v_{2}^{*}\right)<\cdots<d_{r}\left(v, v_{d}^{*}\right)$.

Let $p_{1}, p_{2}, \ldots, p_{d}$ be direct interval paths associated with $I_{v, e, e^{\prime}}$, where $p_{i}=p_{\text {opt }}^{\prime}(s, v)+$ $\overline{v v_{i}^{*}}$ for $1 \leqslant i \leqslant d$. For each $v_{i}^{*}$, let $P_{i}=\left\{p_{j, i} \mid 1 \leqslant j \leqslant d\right\}$, where $p_{j, i}=p_{j}+\overline{v_{j}^{*} v_{i}^{*}}$. Observe that $p_{i} \in P_{i}$ as $p_{i}=p_{i}+\overline{v_{i}^{*} v_{i}^{*}}$. All paths in $P_{i}$ except $p_{i}$ are extended interval paths. $P_{i}$ is the set of all interval paths associated with $I_{v, e, e^{\prime}}$ that connect $s$ and $v_{i}^{*}$. An interval path $p^{\prime} \in P_{i}$ is said to be locally optimal if $\left\|p^{\prime}\right\|=\min \left\{\|p\| \mid p \in P_{i}\right\}$. Obviously, it is only necessary to insert these locally optimal paths into QLIST.

\subsubsection{Picking the next interval path to insert into QLIST}

For each $v_{i}^{*}$, BUSHWHACK inserts into QLIST only one interval path $p_{i}^{*}$, called adopted interval path, that connects $s$ and $v_{i}^{*}$. In the following we describe how BUSHWHACK determines these adopted interval paths $p_{1}^{*}, p_{2}^{*}, \ldots, p_{d}^{*}$ for $I_{v, e, e^{\prime}}$ In Section 5 we will prove that these paths are indeed locally optimal.

If $w_{e^{\prime}}=w_{r}$, for each $v_{i}^{*}$ it is always less costly to travel directly from $v$ to $v_{i}^{*}$ than to first travel from $v$ to some other point on $e^{\prime}$ and then (along edge $e^{\prime}$ ) to $v_{i}^{*}$, and therefore $p_{i}^{*}=p_{i}$.

Now we consider the more interesting case where $w_{e^{\prime}}<w_{r}$. We let $v_{h}$ be the perpendicular point of $v$ on edge $e^{\prime}$ and let $h=\left|\overline{v v_{h}}\right|$. Since $I_{v, e, e^{\prime}}$ is a monotonic interval, $v_{1}^{*}$ is between $v_{h}$ and $v_{d}^{*}$ on $e^{\prime}$, as $v_{1}^{*}$ precedes $v_{d}^{*}$ in $I_{v, e, e^{\prime}}$. Let $u$ be the point on ray $\overrightarrow{v_{h} v_{d}^{*}}$ such that

$$
\frac{\left|\overline{v_{h} u}\right| \cdot w_{r}}{\sqrt{\left|\overline{v_{h} u}\right|^{2}+h^{2}}}=w_{e^{\prime}} .
$$

Therefore, for any point $u^{\prime}$ on ray $\overrightarrow{v_{h} v_{d}^{*}}$, we have 


$$
\begin{aligned}
& \frac{\left|\overline{v_{h} u^{\prime}}\right| \cdot w_{r}}{\sqrt{\left|\overline{v_{h} u^{\prime}}\right|^{2}+h^{2}}}<w_{e^{\prime}} \quad \text { if } u^{\prime} \text { is between } v_{h} \text { and } u, \\
& \frac{\left|\overline{v_{h} u^{\prime}}\right| \cdot w_{r}}{\sqrt{\left|\overline{v_{h} u^{\prime}}\right|^{2}+h^{2}}}>w_{e^{\prime}} \quad \text { if } u^{\prime} \text { is beyond } u .
\end{aligned}
$$

The intuition about the location of $u$ can be explained using an analogy to Snell's Law: $u$ is the critical point on $e^{\prime}$ such that, if an optimal path (i.e., a light ray) originating from $v$ hits $e^{\prime}$ at any point between $v_{h}$ and $u$, it will leave $e^{\prime}$ with a refraction angle less than $\frac{\pi}{2}$; however, if the optimal path hits $e^{\prime}$ at any point beyond $u$, it will travel along $e^{\prime}$ (and thus the refraction angle is $\frac{\pi}{2}$ ).

If $u$ is between $v_{h}$ and $v_{1}^{*}$, each $p_{i}^{*}$ is chosen to be $p_{1, i}$ for each $i, 1 \leqslant i \leqslant d$. That is, each interval path $p_{i}^{*}$ except $p_{1}^{*}$ is an edge-crawling path that extends $p_{1}$ by one segment. If $v_{d}^{*}$ is between $v_{h}$ and $u$, each $p_{i}^{*}$ is chosen to be $p_{i}$. In this case, every interval edge $p_{i}^{*}$ is a face-crossing path that extends $p_{\text {opt }}^{\prime}(s, v)$ by one segment.

Otherwise, $u$ lies between $v_{k}^{*}$ and $v_{k+1}^{*}$ on edge $e^{\prime}$ for some $k, 1 \leqslant k<d$. Then $p_{i}^{*}$ can be determined as follows.

Definition 3. Suppose $v_{k}^{*}$ is the Steiner point inside interval $I_{v, e, e^{\prime}}$ such that $u$, as defined above, is between $v_{k}^{*}$ and $v_{k+1}^{*}$, then

(a) if $1 \leqslant i \leqslant k$, then $p_{i}^{*}$ is defined to be $p_{i}$;

(b) if $i=k+1$, then $p_{i}^{*}$ is defined to be $p_{k+1}$ or $p_{k, k+1}$, whichever is less costly;

(c) if $k+1<i \leqslant d$, then $p_{i}^{*}$ is defined to be $p_{k+1}^{*}+\overline{v_{k+1}^{*} v_{i}^{*}}$.

The propagation process terminates when all points in $I_{v, e, e^{\prime}}$ are reached by such interval paths. Observe that this process may be terminated before $p_{d}^{*}$ is generated and inserted into QLIST. This would occur when another interval $I_{v^{\prime}, e, e^{\prime}}$ is created that re-adjusts the range of $I_{v, e, e^{\prime}}$.

By the BUSHWHACK algorithm, each interval $I_{v, e, e^{\prime}}$ will generate no more than $O\left(d^{\prime}\right)$ locally optimal interval paths, where $d^{\prime}$ is the number of points inside $I_{v, e, e^{\prime}}$ after all points on $e$ are discovered. Thus, for each interval list $\operatorname{ILIST}_{e, e^{\prime}}$, only $O(m)$ interval paths are inserted into QLIST. The total number of interval paths, therefore, is $O(m n)$.

From the propagation scheme of interval paths it is clear that, once an interval $I_{v, e, e^{\prime}}$ is created, at any time in path list QLIST there is exactly one interval path $p$ associated with $I_{v, e, e^{\prime}}$, until the last interval path associated with $I_{v, e, e^{\prime}}$ is extracted from QLIST. We call path $p$ the current interval path of $I_{v, e, e^{\prime}}$ and denote it by $p_{v, e, e^{\prime}}$. The Steiner point in $I_{v, e, e^{\prime}}$ to which path $p_{v, e, e^{\prime}}$ connects is correspondingly referred to as the current Steiner point of $I_{v, e, e^{\prime}}$ and is denoted by $v_{v, e, e^{\prime}}^{*}$. For any point $v^{*}$ preceding $v_{v, e, e^{\prime}}^{*}$ in $I_{v, e, e^{\prime}}$, a locally optimal interval path that connects $s$ and $v^{*}$ has already been extracted from QLIST. We call such a point a processed interval point of $I_{v, e, e^{\prime}}$. Any point $v^{*}$ following $v_{v, e, e^{\prime}}^{*}$ in $I_{v, e, e^{\prime}}$ is an unprocessed interval point. According to the definitions, we can establish the following lemma. 
Lemma 2. For any interval $I_{v, e, e^{\prime}}$, there is always a current Steiner point for $I_{v, e, e^{\prime}}$ in QLIST until all points in $I_{v, e, e^{\prime}}$ are processed.

\subsubsection{Picking non-interval paths to insert into QLIST}

We also need to create two other paths for each interval. Let $v_{0}^{*}$ be the discrete point next to $v_{1}^{*}$ outside interval $I_{v, e, e^{\prime}}$, and let $v^{\prime}$ be the discovered point on $e$ whose interval $I_{v^{\prime}, e, e^{\prime}}$ contains $v_{0}^{*}$, as shown in Fig. 9. We insert path $p_{0}^{*}=p_{1}^{*}+\overline{v_{1}^{*} v_{0}^{*}}$ into QLIST when $I_{v, e, e^{\prime}}$ is created, if $d_{\text {opt }}^{\prime}(s, v)+d_{r}\left(v, v_{1}^{*}\right)+d_{e}\left(v_{1}^{*}, v_{0}^{*}\right)<d_{\text {opt }}^{\prime}\left(s, v^{\prime}\right)+d_{r}\left(v^{\prime}, v_{0}^{*}\right)$. That is, even though to construct a path from $s$ to $v_{0}^{*}$ it is more costly to go directly from $v$ to $v_{0}^{*}$ than to go directly from $v^{\prime}$ to $v_{0}^{*}$ (as $v_{0}^{*}$ is inside $I_{v^{\prime}, e, e^{\prime}}$ ), if traveling on line segment $\overline{v_{1}^{*} v_{0}^{*}}$ along edge $e^{\prime}$ is cost-saving enough, it may be a better way to go from $s$ to $v_{0}^{*}$ through $v$ and then $v_{1}^{*}$ than to go through $v^{\prime}$.

Similarly, we will add path $p_{d+1}^{*}=p_{d}^{*}+\overline{v_{d}^{*} v_{d+1}^{*}}$, if necessary, after all interval paths associated with this interval are inserted. Here $v_{d+1}^{*}$ is the discrete point next to $v_{d}^{*}$ outside $I_{v, e, e^{\prime}}$. We call these two paths non-interval paths as they are not associated with any interval. These non-interval paths will also be propagated when they are removed from QLIST, as we will show later in this paper.

\subsubsection{Continuing path propagation after interval is partially depleted}

Recall that in Section 3.2 we showed that an existing interval $I_{v^{\prime}, e, e^{\prime}}$ could be partially or totally depleted by a newly created interval $I_{v, e, e^{\prime}}$. If $I_{v^{\prime}, e, e^{\prime}}$ is totally depleted, the current interval path $p_{v^{\prime}, e, e^{\prime}}$ no longer needs to be considered as a potential optimal discrete path. We call it an invalid interval path. Unlike other (valid) candidate optimal paths, an invalid interval path will not be propagated when it is extracted from QLIST.

For a partially depleted interval $I_{v^{\prime}, e, e^{\prime}}$, if the current Steiner point $v_{v^{\prime}, e, e^{\prime}}^{*}$ still belongs $I_{v^{\prime}, e, e^{\prime}}$ after the new interval $I_{v, e, e^{\prime}}$ is created, the current interval path $p_{v^{\prime}, e, e^{\prime}}$ of $I_{v^{\prime}, e, e^{\prime}}$ is still locally optimal and we will still propagate it when it is being extracted from QLIST. However, if $v_{v^{\prime}, e, e^{\prime}}^{*}$ is now included in $I_{v, e, e^{\prime}}, p_{v^{\prime}, e, e^{\prime}}$ becomes an invalid interval path. Just as in the case of totally depleted intervals, we will extract $p_{v^{\prime}, e, e^{\prime}}$ from QLIST without propagation when it becomes the least costly path in QLIST.

This will, however, stop the chain of propagation of interval paths associated with $I_{v^{\prime}, e, e^{\prime}}$, as after $p_{v^{\prime}, e, e^{\prime}}$ is extracted from QLIST there will be no interval path in QLIST that is associated with $I_{v^{\prime}, e, e^{\prime}}$. This causes a problem in the case that there are still unprocessed points inside the adjusted $I_{v^{\prime}, e, e^{\prime}}$. To fix this problem, we will treat the adjusted $I_{v^{\prime}, e, e^{\prime}}$ as a newly created interval and insert into QLIST the interval path for the first unprocessed point in $I_{v^{\prime}, e, e^{\prime}}$. This will re-initiate the process of path propagation for the interval.

\section{The BUSHWHACK algorithm}

The BUSHWHACK algorithm maintains three types of dynamic lists:

QLIST the list of candidate optimal paths sorted by path cost.

PLIST $_{e}$ the list of discovered points on edge $e$.

$\operatorname{ILIST}_{e, e^{\prime}}$ the list of intervals for edge $e$ and $e^{\prime}$ that are on the boundary of the same region. 
As we mentioned previously, all paths can be divided into two categories, interval paths and non-interval paths. All face-crossing paths along with some edge-crawling paths are interval paths. We have shown how two non-interval paths are generated for each interval. This section will explain how other non-interval paths are created by extending these paths.

The main body of the BUSHWHACK algorithm is a loop. At each iteration, the candidate optimal path $p$ in QLIST with the minimum cost is extracted from the list. Let $v$ be the ending point of path $p$. If $v$ is not a discovered point (i.e., the distance from $s$ to $v$ is not yet decided), we claim that path $p$ is an optimal discrete path from $s$ to $v$, and $\|p\|$ is the distance from $s$ to $v$.

Here $p_{\text {opt }}^{\prime}(s, s)$ is the zero-cost path from $s$ to $s$.

Procedure HandleNewDiscovery $(v)$ creates new intervals for the newly discovered point $v$, and then inserts into QLIST an interval path associated with each of these intervals.

Whether or not $v$ is a newly discovered point, procedure Propagate $(v, p)$ creates candidate optimal paths by propagating $p$ in a constant number of ways and inserts these paths into QLIST.

Algorithm 1. Main algorithm FindPath(s,t).

1: insert path $p_{\mathrm{opt}}^{\prime}(s, s)$ into QLIST

2: while $t$ is not reached do

3: $\quad$ extract the least costly path $p(s, v)$ from QLIST

4: if $v$ is a not a discovered point then

5: $\quad p_{\text {opt }}^{\prime}(s, v) \leftarrow p(s, v) ; d_{\text {opt }}^{\prime}(s, v) \leftarrow\|p(s, v)\|$

6: $\quad$ HandleNewDiscovery $(v)$

7: $\quad \operatorname{Propagate}(v, p)$

Algorithm 2. Procedure HandleNewDiscovery $(v)$.

1: if $v$ is a Steiner point on an edge $e$ then

2: for all region $r$ incident to $e$ do

3: $\quad$ for all edge $e^{\prime}$ of $r$ that is not $e$ do

4: $\quad$ create interval $I_{v, e, e^{\prime}}$

5: else

6: for all edge $e$ incident to $v$ do

7: $\quad v_{\text {next }} \leftarrow$ the neighboring Steiner point of $v$ on $e$

8: $\quad$ insert path $p_{\text {new }}=p+\overline{v v_{\text {next }}}$ into QLIST

9: $\quad$ for all region $r$ incident to $e$ do

10: $\quad$ for all edge $e^{\prime}$ of $r$ that is not $e$ do

11: $\quad$ create interval $I_{v, e, e^{\prime}}$

12: for all newly created interval $I_{v, e, e^{\prime}} d o$

13: $\quad$ split $I_{v, e, e^{\prime}}$ into monotonic intervals $I_{v, e, e^{\prime}}^{1}$ and $I_{v, e, e^{\prime}}^{2}$

14: for all monotonic interval $I_{v, e, e^{\prime}}^{j}, 1 \leqslant j \leqslant 2 d o$

15: $\quad$ insert into QLIST the first non-interval path for $I_{v, e, e^{\prime}}^{j}$ if necessary

16: $\quad$ insert into QLIST the first interval path for $I_{v, e, e^{\prime}}^{j}$ 
Algorithm 3. Procedure Propagate $(v, p)$.

1: if $p$ is an interval path associated with $I_{v^{\prime}, e^{\prime}, e}$ then

2: if $p$ is still valid then

3: if $v$ is the last point in $I_{v^{\prime}, e^{\prime}, e^{\prime}}$ then

4: $\quad$ insert into QLIST the second non-interval path for $I_{v^{\prime}, e^{\prime}, e^{\prime}}$ if necessary

5: $\quad$ else

6: $\quad$ insert into QLIST the next interval path for $I_{v^{\prime}, e^{\prime}, e}$

7: else

8: $\quad e \leftarrow$ the boundary edge on which the last segment of $p$ is lying

9: if $v$ is not an endpoint of $e$ then

10: $\quad v_{\text {prev }} \leftarrow$ the previous point of $v$ on path $p$

11: $\quad v_{\text {next }} \leftarrow$ the neighboring point of $v$ that is not between $v_{\text {prev }}$ and $v$ then

12: if there has not already been a non-interval edge-crawling path that extends to $v_{\text {next }}$ from $v$ then

13: $\quad$ insert path $p_{\text {new }}=p+\overline{v v_{\text {next }}}$ into QLIST

We have explained previously how paths are propagated inside intervals. Observe that the task of handling interval paths is accomplished by the combination of the procedures Propagate and HandleNewDiscovery. For example, intervals are created in the procedure HandleNewDiscovery when a point $v$ is discovered. At the same time, the first interval path associated with each new interval is inserted into QLIST (line 16 of the procedure HandleNewDiscovery). The propagation of interval paths for each interval is accomplished in the procedure Propagate (line 6).

In Section 3.4.3 we specified that each interval would also generate two non-interval paths. One of them is generated when the interval is created (line 15 of the procedure HandleNewDiscovery), while the other is generated when the last interval path of that interval is extracted from QLIST (line 4 of the procedure Propagate). Another situation that generates non-interval paths is when the newly discovered point $v$ is a vertex. In this case, for each incident boundary edge $e$ of $v$ a non-interval path is inserted into QLIST; this path extends $p_{\text {opt }}^{\prime}(s, v)$ to the neighboring Steiner point of $v$ along $e$, as indicated by line 8 of HandleNewDiscovery.

All the non-interval paths are edge-crawling paths. According to the procedure Propagate (line 8 to line 13), when an non-interval path $p$ from $s$ to $v$ is extracted from QLIST, we may insert an extension of this path into QLIST. Suppose $\overline{v_{\text {prev }} v}$ is the last segment of path $p$. Since $p$ is edge-crawling, $v_{\text {prev }}$ is on the same edge $e$ as $v$. Let $v_{\text {next }}$ be the adjacent Steiner point of $v$ on $e$ that is on the other side of $v_{\text {prev }}$. We insert path $p+\overline{v v_{\text {next }}}$ into QLIST, if there has not been another path $p^{\prime}+\overline{v v_{\text {next }}}$ inserted into QLIST.

It is easy to see that, for each Steiner point $v \in V_{s}$, there will be at most two non-interval paths from $s$ to $v$ inserted into QLIST, one approaching $v$ from left and one approaching $v$ from right. Similarly, for any vertex $v \in V$, there will be at most $D(v)$ non-interval paths that connect $s$ and $v$, one from each edge incident to $v$. Here $D(v)$ is the number of incident boundary edges of $v$ in the original triangular decomposition. Thus, the total number of non-interval paths is bounded by $\sum_{v \in V} D(v)+\sum_{e \in E} 2 m=2|E|+2 m|E|=O(m n)$. The total number of all paths inserted into QLIST, therefore, is bounded by $O(\mathrm{mn})$. 


\section{Complexity and correctness}

\subsection{Complexity}

The complexity of the BUSHWHACK algorithm depends on three factors:

Cost of maintaining QLIST: There are $O(m n)$ candidate optimal paths inserted into QLIST. Each insertion or deletion operation takes $O(\log (m n))$ time. The total cost of maintaining QLIST is $O(\mathrm{~nm} \log \mathrm{nm})$.

Cost of maintaining PLIST $_{e}$ : There are $O(m)$ points on each edge. Each insertion or deletion operation takes $O(\log m)$ time. As there are $O(n)$ boundary edges, the total cost of maintaining $\mathrm{PLIST}_{e}$ for all $e$ is $O(n m \log m)$.

Cost of maintaining ILIST ${\text { I }, e^{\prime}}$ : When a point $v$ on $e$ is discovered, we first need to decide the range of $I_{v, e, e^{\prime}}$ for each $e^{\prime}$ that share a region with $e$. Deciding each of the two endpoints of $I_{v, e, e^{\prime}}$ can be done by a binary search on intervals in $\operatorname{ILIST}_{e, e^{\prime}}$. As the maximum number of intervals in $\operatorname{ILIST}_{e, e^{\prime}}$ is $m$, a binary search takes $O(\log m)$ time. Also, the time to insert an interval to the interval list $\operatorname{ILIST}_{e, e^{\prime}}$ is $O(\log m)$. As there are $O(n)$ interval lists, the total cost of maintaining these interval lists is $O(n m \log m)$.

The complexity of the algorithm, therefore, is $O(n m \log n m)$.

In Section 2 we claimed that, in average, for each Steiner point $v$ BUSHWHACK needs to evaluate the costs of only $O(\log m)$ adjacent edges of $v$. Even though inside each region only $O(\mathrm{~m})$ edges are ever used by candidate optimal paths inserted into QLIST, that is, $O(1)$ edges per Steiner point in the region, BUSHWHACK has to evaluate the costs of additional edges in order to maintain the intervals. As mentioned above, deciding the bounding points of a new interval $I_{v, e, e^{\prime}}$ takes a binary search of $O(\log m)$ steps. At each step, BUSHWHACK needs to compare the cost of $\overline{v v^{*}}$ for some $v^{*} \in e^{\prime}$ with the cost of $\overline{v^{\prime} v^{*}}$, where $v^{\prime}$ is one of the two neighboring discovered points of $v$ on $e$. As a result, $O(\log m)$ edges are evaluated for each Steiner point.

\subsection{Correctness}

To show that the BUSHWHACK algorithm is correct, it suffices to prove the following theorem.

Theorem 1. When path $p(s, v)$ is extracted from QLIST, if $v$ is not yet discovered, $p(s, v)$ is an optimal discrete path.

Before we give the proof of Theorem 1, we first establish the following lemma.

Lemma 3. Each adopted interval path $p_{i}^{*}$ as defined in Section 3.4.2 is locally optimal.

Proof. The proof for the case where $w_{e^{\prime}}=w_{r}$ is trivial. In the following we assume that $w_{e^{\prime}}<w_{r}$. Further, we assume that the point $u$ on ray $\overrightarrow{v_{h} v_{d}^{*}}$ such that

$$
\frac{\left|\overline{v_{h} u}\right| \cdot w_{r}}{\sqrt{\left|\overline{v_{h} u}\right|^{2}+h^{2}}}=w_{e^{\prime}}
$$


lies between $v_{k}^{*}$ and $v_{k+1}^{*}$ for some $k, 1 \leqslant k<d$, as shown in Fig. 9. The other two cases can be proved similarly. We need to show that for each $i$, the adopted interval path $p_{i}^{*}$ is locally optimal.

We first discuss the case in which $i \leqslant k$ and therefore, according to Definition 3(a), $p_{i}^{*}=p_{i}$. For any $j$, if $j \geqslant i,\left\|p_{j, i}\right\|=\left\|p_{j}\right\|+d_{e}\left(v_{j}^{*}, v_{i}^{*}\right) \geqslant\left\|p_{j}\right\| \geqslant\left\|p_{i}\right\|=\left\|p_{i}^{*}\right\|$. If $j<i$, we have the following inequality:

$$
\begin{aligned}
\left\|p_{i}^{*}\right\|=\left\|p_{i}\right\| & =\left\|p_{j}\right\|+w_{r} \cdot\left(\sqrt{\left|\overline{v_{h} v_{i}^{*}}\right|^{2}+h^{2}}-\sqrt{\left|\overline{v_{h} v_{j}^{*}}\right|^{2}+h^{2}}\right) \\
& =\left\|p_{j}\right\|+\int_{\left|\overline{v_{h} v_{j}^{*}}\right|}^{\left|\overline{v_{h} v_{i}^{*}}\right|} w_{r} \mathrm{~d}\left(\sqrt{x^{2}+h^{2}}\right)=\left\|p_{j}\right\|+\int_{\left|\overline{v_{h} v_{j}^{*}}\right|} \frac{w_{r} \cdot x}{\sqrt{x^{2}+h^{2}}} \mathrm{~d} x \\
& <\left\|p_{j}\right\|+\int_{\left|\overline{v_{h} v_{i}^{*}}\right|} w_{e^{\prime}} \mathrm{d} x \quad \text { (by Eq. (1)) } \\
& =\left\|p_{j}\right\|+w_{e^{\prime}} \cdot\left|\overline{v_{j}^{*} v_{i}^{*}}\right|=\left\|p_{j, i}\right\| .
\end{aligned}
$$

Now we consider the case in which $i=k+1$ and therefore $\left\|p_{i}^{*}\right\|=\min \left\{\left\|p_{k, k+1}\right\|\right.$, $\left.\left\|p_{k+1}\right\|\right\}$. For any $j>i$,

$$
\left\|p_{j, i}\right\| \geqslant\left\|p_{i}\right\|=\left\|p_{i, i}\right\| \geqslant\left\|p_{i}^{*}\right\| .
$$

For any $j<i$,

$$
\left\|p_{j, i}\right\|=\left\|p_{j, k}\right\|+w_{e^{\prime}} \cdot\left|\overline{v_{k}^{*} v_{k+1}^{*}}\right| \geqslant\left\|p_{k}^{*}\right\|+w_{e^{\prime}} \cdot\left|\overline{v_{k}^{*} v_{k+1}^{*}}\right|=\left\|p_{k, k+1}\right\| \geqslant p_{i}^{*} .
$$

Finally, we suppose $k+1<i \leqslant d$. For any $j \leqslant k+1$,

$$
\left\|p_{j, i}\right\|=\left\|p_{j, k+1}\right\|+w_{e^{\prime}} \cdot\left|\overline{v_{k+1}^{*} v_{i}^{*}}\right| \geqslant\left\|p_{k+1}^{*}\right\|+w_{e^{\prime}} \cdot\left|\overline{v_{k+1}^{*} v_{i}^{*}}\right|=\left\|p_{i}^{*}\right\| .
$$

For any $j \geqslant i$,

$$
\left\|p_{j, i}\right\|=\left\|p_{j}\right\|+d_{e}\left(v_{j}^{*}, v_{i}^{*}\right) \geqslant\left\|p_{j}\right\| \geqslant\left\|p_{i}\right\| \geqslant\left\|p_{i}^{*}\right\| .
$$

For any $j, k+1<j \leqslant i$, we have the following inequality:

$$
\begin{aligned}
\left\|p_{i}^{*}\right\| & =\left\|p_{k+1}^{*}\right\|+w_{e^{\prime}} \cdot\left|\overline{v_{k+1}^{*} v_{j}^{*}}\right|+w_{e^{\prime}} \cdot\left|\overline{v_{j}^{*} v_{i}^{*}}\right| \\
& =\left\|p_{k+1}^{*}\right\|+\int_{\left|\overline{v_{h} v_{j}^{*}}\right|} w_{e^{\prime}} \mathrm{d} x+w_{e^{\prime}} \cdot\left|\overline{v_{j}^{*} v_{i}^{*}}\right| \\
& <\left\|p_{k+1}^{*}\right\|+\int_{\left|\overline{v_{h} v_{k+1}^{*}}\right|}^{\left|\overline{v_{h} v_{j}^{*}}\right|} \frac{w_{r} \cdot x}{\sqrt{x^{2}+h^{2}}} \mathrm{~d} x+w_{e^{\prime}} \cdot\left|\overline{v_{j}^{*} v_{i}^{*}}\right| \quad \text { (by Eq. (2)) }
\end{aligned}
$$




$$
\begin{aligned}
& =\left\|p_{k+1}\right\|+w_{r} \cdot \int_{\left|\overline{v_{h} v_{k+1}^{*}}\right|}^{\left|\overline{v_{h} v_{j}^{*}}\right|} \mathrm{d}\left(\sqrt{x^{2}+h^{2}}\right)+w_{e^{\prime}} \cdot\left|\overline{v_{j}^{*} v_{i}^{*}}\right| \\
& =\left\|p_{k+1}\right\|+w_{r} \cdot\left(\sqrt{\left|\overline{v_{h} v_{j}^{*}}\right|^{2}+h^{2}}-\sqrt{\left|\overline{v_{h} v_{k+1}^{*}}\right|^{2}+h^{2}}\right)+w_{e^{\prime}} \cdot\left|\overline{v_{j}^{*} v_{i}^{*}}\right| \\
& =\left\|p_{j}\right\|+w_{e^{\prime}} \cdot\left|\overline{v_{j}^{*} v_{i}^{*}}\right|=\left\|p_{j, i}\right\| .
\end{aligned}
$$

This finishes the proof.

In a sense, each locally optimal paths is the "discrete version" of the optimal path defined by Snell's Law, as the true optimal path may not necessarily bend at a Steiner point on $e^{\prime}$.

Now we are ready to prove the main theorem. The proof is analogous to the correctness proof of Dijkstra's algorithm. The main difference lies in the fact that BUSHWHACK employs a lazy and best-first path propagation scheme, and therefore we need to prove that BUSHWHACK still can find an optimal discrete path despite avoiding using most of the graph edges in $\mathcal{G}$. More specifically, we need to show that for any discrete point $v$, before any non-optimal discrete path $p^{\prime}$ connecting $s$ and $v$ is removed from QLIST, an optimal discrete path $p^{*}$ connecting $s$ and $v$ must have been inserted into QLIST, and thus will be removed from QLIST before $p^{\prime}$ is.

Proof of Theorem 1. For the sake of contradiction we assume that such a path $p$ is not optimal. Let $p^{*}$ be an optimal discrete path from $s$ to $v$ such that $\left\|p^{*}\right\|<\|p\|$, as shown in Fig. 10. Let $u_{\text {next }}$ be the first undiscovered point on $p^{*}$ and let $u$ be the predecessor of $u_{\text {next }}$ on $p^{*}$. As $p^{*}$ is an optimal discrete path from $s$ to $v, p^{*}\left[s, u_{\text {next }}\right]$ must be an optimal discrete path from $s$ to $u_{\text {next }}$, and $p^{*}[s, u]$ must be an optimal discrete path from $s$ to $u$.

There are two cases. We first consider the case when segment $\overline{u u_{\text {next }}}$ is face-crossing. Let $r$ be the region in which $\overline{u u_{\text {next }}}$ lies. Let $e$ and $e^{\prime}$ be the boundary edges of $r$

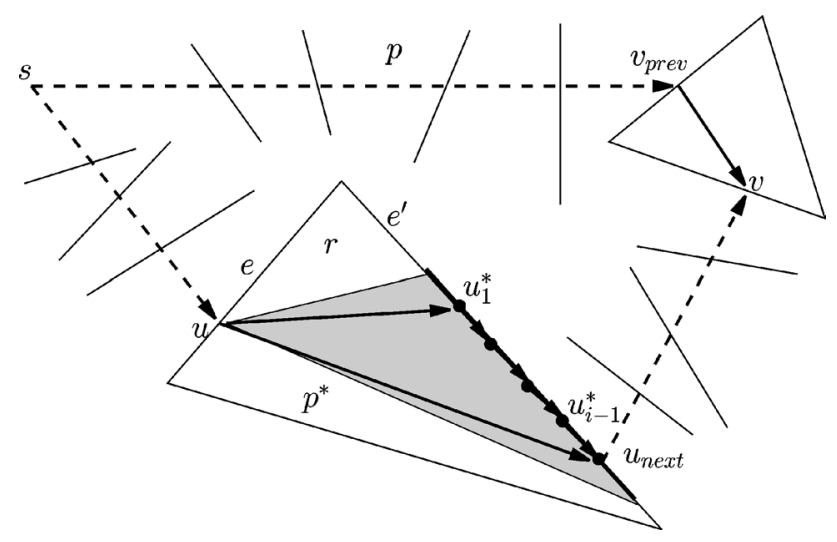

Fig. 10. Correctness proof. 
such that $u \in e$ and $u_{\text {next }} \in e^{\prime}$. Since $u$ is a discovered point, $I_{u, e, e^{\prime}}$ has already been created and inserted into $\operatorname{ILIST}_{e, e^{\prime}}$. According to Definition $1, u_{\text {next }}$ must be inside interval $I_{u, e, e^{\prime}}$. Otherwise there should be anther discovered point $u^{\prime}$ on $e$ such that $d_{\text {opt }}^{\prime}\left(s, u^{\prime}\right)+d_{r}\left(u^{\prime}, u_{\text {next }}\right)<d_{\text {opt }}^{\prime}(s, u)+d_{r}\left(u, u_{\text {next }}\right)=\left\|p^{*}\left[s, u_{\text {next }}\right]\right\|$. This is a contradiction to the fact that $p^{*}\left[s, u_{\text {next }}\right]$ is an optimal discrete path from $s$ to $u_{\text {next }}$. As $u_{\text {next }}$ is not discovered yet, $u_{\text {next }}$ must have not been processed. Therefore, according to Lemma 2 there is a current Steiner point $v_{u, e, e^{\prime}}^{*}$ for $I_{u, e, e^{\prime}}$, and $v_{u, e, e^{\prime}}^{*}$ is ahead of $u_{\text {next }}$ in interval $I_{u, e . e^{\prime}}$. Let $u_{1}^{*}, u_{2}^{*}, \ldots, u_{j-1}^{*}, u_{j}^{*}=v_{u, e, e^{\prime}}^{*}, u_{j+1}^{*}, \ldots, u_{i-1}^{*}, u_{i}^{*}=u_{\text {next }}, u_{i+1}^{*}, \ldots, u_{d}$ be the $d$ Steiner points in interval $I_{u, e, e^{\prime}}$. For each $k, 1 \leqslant k \leqslant d$, let $p_{k}^{*}$ be the adopted interval path chosen for $u_{k}^{*}$. According to Lemma 3 , these paths are locally optimal. In particular, $p_{j}^{*}$ is the current interval path of $I_{u, e, e^{\prime}}$ as it connects $s$ and $u_{j}^{*}$, the current Steiner point. As $\left\|p_{j}^{*}\right\| \leqslant\left\|p_{i}^{*}\right\|=\left\|p^{*}\left[s, u_{\text {next }}\right]\right\| \leqslant\left\|p^{*}\right\|<\|p\|, p_{j}^{*}$ must be extracted from QLIST before $p$ is extracted from QLIST. For the same reason, any $p_{k}^{*}, j \leqslant k \leqslant i$, must be extracted from QLIST before $p$. This is a contradiction to the assumption that $u_{\text {next }}$ is undiscovered at the time $p$ is extracted from QLIST.

Now we consider the case when $\overline{u u_{\text {next }}}$ is edge-crawling. Let $e$ be the edge that contains both $u$ and $u_{\text {next }}$. We first assume that $u \in V$. Let $u_{1}=u, u_{2}, \ldots, u_{i-1}, u_{i}=u_{\text {next }}$ be the Steiner points from $u$ to $u_{\text {next }}$ on $e$. According to line 8 of procedure HandleNewDiscovery, path $p_{\text {opt }}^{\prime}(s, u)+\overline{u u_{2}}$ is inserted into QLIST when $u$ is discovered. As $\left\|p_{\text {opt }}^{\prime}(s, u)+\overline{u u_{2}}\right\|=$ $\left\|p_{\text {opt }}^{\prime}(s, u)\right\|+d_{e}\left(u, u_{2}\right)<\left\|p_{\text {opt }}^{\prime}(s, u)\right\|+d_{e}\left(u, u_{i}\right)=\left\|p^{*}\left[s, u_{\text {next }}\right]\right\| \leqslant\left\|p^{*}\right\|<\|p\|$, path $p_{\text {opt }}^{\prime}(s, u)+\overline{u u_{2}}$ should be extracted from QLIST before $p$ is. Similarly, we can show that for each $k, 1<k \leqslant i$, path $p_{\mathrm{opt}}^{\prime}(s, u)+\overline{u u_{k}}$ is extracted from QLIST before $p$ is. This is a contradiction to the assumption that $u_{\text {next }}$ is not discovered when $p$ is extracted from QLIST.

Next we assume that $u \in V_{s}$. Let $u_{\text {prev }}$ be the predecessor of $u$ on path $p^{*}$. Then $u_{\text {prev }}$ must not be on $e$ and thus segment $\overline{u_{\text {prev }} u}$ is face-crossing. Let $e_{\text {prev }}$ be an edge that contains $u_{\text {prev }}$. As $u_{\text {prev }}$ is a discovered point, interval $I_{u_{\text {prev }}, e_{\text {prev }}, e}$ must have been created and inserted into ILIST $e_{e_{\text {prev }}, e} . u$ must be inside $I_{u_{\mathrm{prev}}, e_{\mathrm{prev}}, e}$ as otherwise $p^{*}[s, u]$ is not an optimal discrete path.

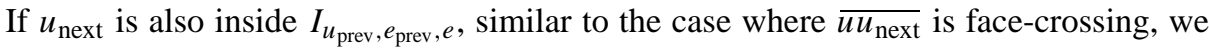
can prove that a locally optimal path associated with $I_{u_{\mathrm{prev}}, e_{\mathrm{prev}}, e}$ that connects $s$ and $u_{\text {next }}$ must have been extracted from $I_{u_{\text {prev }}, e_{\text {prev }}, e}$ before $p$ is. Again a contradiction.

If $u_{\text {next }}$ is not in the interval, let $u_{1}^{*}, u_{2}^{*}, \ldots, u_{i-1}^{*}, u_{i}^{*}=u, u_{i+1}^{*}, \ldots, u_{d}^{*}$ be the points

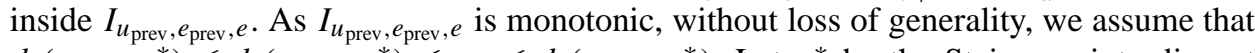
$d_{r}\left(u_{\text {prev }}, u_{1}^{*}\right) \leqslant d_{r}\left(u_{\text {prev }}, u_{2}^{*}\right) \leqslant \cdots \leqslant d_{r}\left(u_{\text {prev }}, u_{d}^{*}\right)$. Let $u_{0}^{*}$ be the Steiner point adjacent

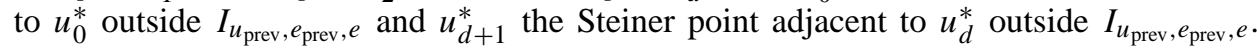
If $u_{d}$ is between $u$ and $u_{\text {next }}$, as $\left\|p_{d}^{*}\right\| \leqslant\left\|p^{*}[s, u]+\overline{u u_{d}^{*}}\right\|=\left\|p^{*}[s, u]\right\|+d_{e}\left(u, u_{d}^{*}\right)<$ $\left\|p^{*}[s, u]\right\|+d_{e}\left(u, u_{\text {next }}\right)=\left\|p^{*}\left[s, u_{\text {next }}\right]\right\| \leqslant\left\|p^{*}\right\|<\|p\|$, all locally optimal paths associated with $I_{u_{\text {prev }}, e_{\text {prev }}, e}$ must be extracted from QLIST before $p$ is extracted. Therefore, according to line 4 of procedure Propagate, non-interval path $p_{d+1}^{*}=p_{d}^{*}+\overline{u_{d}^{*} u_{d+1}^{*}}$ must be inserted into QLIST before $p$ is extracted from QLIST. This non-interval path, also less costly than $p$, is eventually going to be extended along $e$ to $u_{\text {next }}$ before $p$ is extracted, a contradiction to the fact that $u_{\text {next }}$ is undiscovered. The case when $u_{1}^{*}$ is between $u$ and $u_{\text {next }}$ can be handled similarly. 
By the above analysis, we show that in any case $u_{\text {next }}$ cannot be an undiscovered point when $p$ is extracted from QLIST. Therefore, such a $p^{*}$ does not exist and hence $p$ is an optimal discrete path from $s$ to $v$.

The above correctness proof may help to explain why we need to propagate the paths the way described by the pseudo code.

\section{Discretization with reduced size}

In this section we describe an improvement of the discretization scheme proposed by Aleksandrov et al. [3]; this improvement removes the dependency of the discretization size (and hence the time complexity of the approximation algorithm) on the unit weight ratio $\mu$.

We first introduce some notations. For any point $v$, we let $E(v)$ be the set of edges incident to $v$ and let $D_{v}$ be the minimum distance between $v$ and edges in $E \backslash E(v)$. For each edge $e \in E$, we let $D_{e}=\sup \left\{D_{v} \mid v \in e\right\}$ and let $v_{e}$ be the point on $e$ so that $D_{v_{e}}=D_{e}$. For each vertex $v$ of the triangular subdivision, the radius $r^{\prime}(v)$ of $v$ is defined to be $\frac{D_{v}}{5}$, and the weighted radius $r(v)$ of $v$ is defined to be $\frac{w_{\min }(v)}{w_{\max }(v)} \cdot r^{\prime}(v)$, where $w_{\min }(v)$ and $w_{\max }(v)$ are the minimum and maximum unit weights among all regions incident to $v$, respectively.

According to the discretization scheme of Aleksandrov et al. [3], for each boundary edge $e=\overline{v_{1} v_{2}}$, the Steiner points on $e$ are chosen as the following. Each vertex $v_{i}$ has a "vertex-vicinity" $S\left(v_{i}\right)$ of radius $r_{\varepsilon}\left(v_{i}\right)=\varepsilon r\left(v_{i}\right)$ and the Steiner points $v_{i, 1}, v_{i, 2}, \ldots, v_{i, k_{i}}$ are placed on the segment of $e$ outside the vertex-vicinities so that $\left|\overline{v_{i} v_{i, 1}}\right|=r_{\varepsilon}\left(v_{i}\right)$, $\left|\overline{v_{i, j} v_{i, j+1}}\right|=\varepsilon D_{v_{i, j}}$ and $\overline{v_{i, k_{i}} v_{i}}+\varepsilon D_{v_{i, k_{i}}} \geqslant\left|\overline{v_{i} v_{e}}\right|$. The number of Steiner points placed on $e$ can be bounded by $C(e) \cdot \frac{1}{\varepsilon} \log \frac{1}{\varepsilon}$, where

$$
C(e)=O\left(\frac{|e|}{D_{e}} \log \frac{|e|}{\sqrt{r\left(v_{1}\right) r\left(v_{2}\right)}}\right)=O\left(\frac{|e|}{D_{e}}\left(\log \frac{|e|}{\sqrt{r^{\prime}\left(v_{1}\right) r^{\prime}\left(v_{2}\right)}}+\log \mu\right)\right) .
$$

This discretization can guarantee a $3 \varepsilon$-good approximate optimal path.

Observe that, for this discretization scheme, on each boundary edge $e$ Steiner points are placed more densely in the portion of $e$ closer to the two endpoints, with the exception that no Steiner point is placed inside the vertex-vicinities. Therefore, the larger the vertex vicinities are, the less Steiner points the discretization needs to use. In the following we show that the radius $r_{\varepsilon}(v)$ of the vertex-vicinity of $v$ can be increased to $\varepsilon r^{\prime}(v)$ while still guaranteeing the same error bound. Here we assume that $\varepsilon \leqslant \frac{1}{2}$.

A piecewise linear path $p$ is said to be a normalized path if it does not cross region boundaries inside vertex vicinities other than at the vertices. That is, for each bending point $u$ of $p$, if $u$ is located on boundary edge $e=\overline{v_{1} v_{2}}$, then either $u$ is one of the endpoints of $e$, or $\left|\overline{v_{i} u}\right| \geqslant r_{\varepsilon}\left(v_{i}\right)$ for $i=1,2$. For example, the path shown in Fig. 11 is not a normalized path, as it passes through $u_{1}$ and $u_{2}$, both of which are inside the vertex vicinity of $v$. We first prove the following lemma.

Lemma 4. For any path $p$ from $s$ to $t$, there is a normalized path $\hat{p}$ from $s$ to $t$ so that $\|\hat{p}\|=\left(1+\frac{\varepsilon}{2}\right) \cdot\|p\|$. 


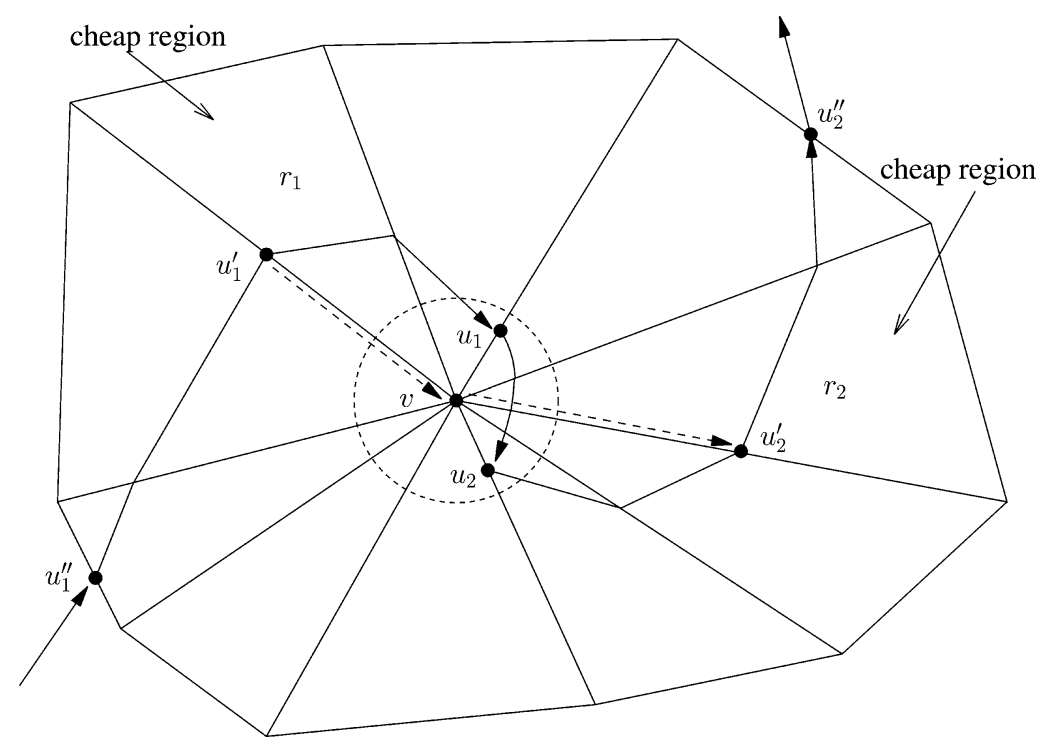

Fig. 11. Path passing through vicinity of a vertex.

Proof. Suppose path $p$ passes through the vertex vicinity $S(v)$ of $v$, as shown in Fig. 11 . We use $u_{1}$ ( $u_{2}$, respectively) to denote the first (last, respectively) bending point of $p$ inside $S(v)$, and use $u_{1}^{\prime \prime}\left(u_{2}^{\prime \prime}\right)$ to denote the first (last, respectively) bending point of $p$ on the boundary of the union of all regions incident to $v$. By the definition of $D_{v}$, we have $\left|p\left[u_{1}^{\prime \prime}, u_{1}\right]\right|+\left|\overline{u_{1} v}\right| \geqslant D_{v}$ and $\left|p\left[u_{2}, u_{2}^{\prime \prime}\right]\right|+\left|\overline{v u_{2}}\right| \geqslant D_{v}$. Therefore,

$$
\left|\overline{u_{1} v}\right| /\left|p\left[u_{1}^{\prime \prime}, u_{1}\right]\right| \leqslant \frac{\varepsilon \cdot D_{v} / 5}{D_{v}-\varepsilon \cdot D_{v} / 5}=\frac{\varepsilon}{5-\varepsilon} \leqslant \frac{\varepsilon}{4},
$$

as $\left|\overline{u_{1} v}\right| \leqslant \frac{\varepsilon D_{v}}{5}$. Similarly, we can prove that $\left|\overline{v u_{2}}\right| /\left|p\left[u_{2}, u_{2}^{\prime \prime}\right]\right| \leqslant \frac{\varepsilon}{4}$.

We let $r_{1}$ be the region with the minimum unit weight among all regions crossed by subpath $p\left[u_{1}^{\prime \prime}, u_{1}\right]$, and $u_{1}^{\prime}$ be the point where $p\left[u_{1}^{\prime \prime}, u_{1}\right]$ enters region $r_{1}$ for the first time. Similarly, we let $r_{2}$ be the region with the minimum unit weight among all regions crossed by subpath $p\left[u_{2}, u_{2}^{\prime \prime}\right]$, and let $u_{2}^{\prime}$ be the point where $p\left[u_{2}, u_{2}^{\prime \prime}\right]$ leaves region $r_{1}$ for the last time.

Consider replacing subpath $p\left[u_{1}^{\prime \prime}, u_{2}^{\prime \prime}\right]$ by normalized subpath $\hat{p}\left[u_{1}^{\prime \prime}, u_{2}^{\prime \prime}\right]=p\left[u_{1}^{\prime \prime}, u_{1}^{\prime}\right]+$ $\overline{u_{1}^{\prime} v}+\overline{v u_{2}^{\prime}}+p\left[u_{2}^{\prime}, u_{2}^{\prime \prime}\right]$. We have the following inequality:

$$
\begin{aligned}
& \left\|\hat{p}\left[u_{1}^{\prime \prime}, u_{2}^{\prime \prime}\right]\right\|-\left\|p\left[u_{1}^{\prime \prime}, u_{2}^{\prime \prime}\right]\right\| \\
& \quad=w_{r_{1}} \cdot\left|\overline{u_{1}^{\prime} v}\right|+w_{r_{2}} \cdot\left|\overline{v u_{2}^{\prime}}\right|-\left\|p\left[u_{1}^{\prime}, u_{1}\right]\right\|-\left\|p\left[u_{1}, u_{2}\right]\right\|-\left\|p\left[u_{2}, u_{2}^{\prime}\right]\right\| \\
& \quad \leqslant\left(w_{r_{1}} \cdot\left|\overline{u_{1}^{\prime} v}\right|-\left\|p\left[u_{1}^{\prime}, u_{1}\right]\right\|\right)+\left(w_{r_{2}} \cdot\left|\overline{v u_{2}^{\prime}}\right|-\left\|p\left[u_{2}, u_{2}^{\prime}\right]\right\|\right) \\
& \quad \leqslant w_{r_{1}}\left(\left|\overline{u_{1}^{\prime} v}\right|-\left|p\left[u_{1}^{\prime}, u_{1}\right]\right|\right)+w_{r_{2}}\left(\left|\overline{v u_{2}^{\prime}}\right|-\left|p\left[u_{2}, u_{2}^{\prime}\right]\right|\right) \\
& \quad \leqslant w_{r_{1}} \cdot\left|\overline{u_{1} v}\right|+w_{r_{2}} \cdot\left|\overline{v u_{2}}\right|
\end{aligned}
$$




$$
\begin{aligned}
& \leqslant w_{r_{1}} \cdot \frac{\varepsilon \cdot\left|p\left[u_{1}^{\prime \prime}, u_{1}\right]\right|}{4}+w_{r_{2}} \cdot \frac{\varepsilon \cdot\left|p\left[u_{2}, u_{2}^{\prime \prime}\right]\right|}{4} \\
& \leqslant \frac{\varepsilon}{4} \cdot\left(\left\|p\left[u_{1}^{\prime \prime}, u_{1}\right]\right\|+\left\|p\left[u_{2}, u_{2}^{\prime \prime}\right]\right\|\right) \\
& \leqslant \frac{\varepsilon}{4} \cdot\left\|p\left[u_{1}^{\prime \prime}, u_{2}^{\prime \prime}\right]\right\| .
\end{aligned}
$$

Therefore, $\left\|\hat{p}\left[u_{1}^{\prime \prime}, u_{2}^{\prime \prime}\right]\right\| \leqslant\left(1+\frac{\varepsilon}{4}\right)\left\|p\left[u_{1}^{\prime \prime}, u_{2}^{\prime \prime}\right]\right\|$. Suppose $p$ passes through $k$ vertex vicinities, $S\left(v_{1}\right), S\left(v_{2}\right), \ldots, S\left(v_{k}\right)$. For each $v_{i}$, we replace the subpath $p_{i}$ of $p$ that passes through $S\left(v_{i}\right)$ by a normalized subpath $\hat{p}_{i}$ as we described above. Let $\hat{p}$ be the resulting normalized path. Noting that the sum of the weighted lengths of $p_{1}, p_{2}, \ldots, p_{k}$ is less than twice the weighted length of $p$, we have $\|\hat{p}\| \leqslant\|p\|+\frac{\varepsilon}{4} \sum_{i=1}^{k}\left\|p_{i}\right\| \leqslant\left(1+\frac{\varepsilon}{2}\right)\|p\|$.

With Lemma 4, it is easy to prove the claimed error bound for this modified discretization:

Theorem 2. The discretization constructed with $r_{\varepsilon}(v)=\varepsilon r^{\prime}(v)$ contains a $3 \varepsilon$-good approximation for an optimal path $p_{\mathrm{opt}}$ from $s$ to $t$, for any vertices $s$ and $t$.

Proof. We first construct a normalized path $\hat{p}$ such that $\|\hat{p}\| \leqslant\left(1+\frac{\varepsilon}{2}\right)\left\|p_{\text {opt }}\right\|$.

We call a segment of a boundary edge bounded by two adjacent discrete points (Steiner points or vertices) a Steiner segment. In particular, if both of the two end points of a Steiner segment are Steiner points, we say that it is a true Steiner segment. Otherwise, if one of the end point is a vertex, we say that the Steiner segment is a half Steiner segment.

For each segment $\overline{v_{1} v_{2}}$ of $\hat{p}$, let $\overline{u_{1,1} u_{1,2}}\left(\overline{u_{2,1} u_{2,2}}\right.$, respectively) be the Steiner segment that contains $v_{1}\left(v_{2}\right.$, respectively). Since $\hat{p}$ is normalized, there are only three cases regarding these two Steiner segments:

(i) both $\overline{u_{1,1} u_{1,2}}$ and $\overline{u_{2,1} u_{2,2}}$ are pure Steiner segments;

(ii) one of $\overline{u_{1,1} u_{1,2}}$ and $\overline{u_{2,1} u_{2,2}}$ is a pure Steiner segment, and the other is a half Steiner segment;

(iii) both $\overline{u_{1,1} u_{1,2}}$ and $\overline{u_{2,1} u_{2,2}}$ are half Steiner segments; however, they do not share the same vertex.

For each of the three cases, it is easy to show that $\left|\overline{u_{1, i} u_{2, j}}\right| \leqslant(1+2 \varepsilon) \cdot\left|\overline{v_{1} v_{2}}\right|$ for any $i=1,2$ and $j=1,2$. So we can construct a discrete path $p^{\prime}$ such that $\left\|p^{\prime}\right\| \leqslant$ $(1+2 \varepsilon)\|\hat{p}\|$. Therefore, $\left\|p^{\prime}\right\| \leqslant(1+2 \varepsilon)\left(1+\frac{\varepsilon}{2}\right)\left\|p_{\text {opt }}\right\|=\left(1+\frac{5}{2} \varepsilon+\varepsilon^{2}\right)\left\|p_{\text {opt }}\right\| \leqslant$ $(1+3 \varepsilon)\left\|p_{\text {opt }}\right\|$, assuming $\varepsilon \leqslant \frac{1}{2}$.

With the modification on the radius for each vertex vicinity, for each edge $e$ the number of Steiner points placed on $e$ is reduced to $C^{\prime}(e) \cdot \frac{1}{\varepsilon} \cdot \log \frac{1}{\varepsilon}$, where $C^{\prime}(e)=$ $O\left(\frac{|e|}{D_{e}} \log \frac{|e|}{\sqrt{r^{\prime}\left(v_{1}\right) r^{\prime}\left(v_{2}\right)}}\right) . C^{\prime}(e)$ is independent of the unit weight ratio, and therefore so is the time complexity of any approximation algorithm that uses this discretization. It appears that each of the previous $\varepsilon$-approximation algorithms has a time complexity dependent on the unit weight ratio. 


\section{Experimental results}

In order to provide a performance comparison, we implemented using Java the following three algorithms:

(1) the BUSHWHACK algorithm, the Algorithm 1 we presented in Section 4;

(2) the pure Dijkstra's algorithm, which searches every incident edge of a discrete point in $\mathcal{G}_{\varepsilon}$;

(3) the cone-based Dijkstra's algorithm (by Aleksandrov et al. [3]), which searches an incident edge of a discrete point only if the edge is inside the geodesic cone of the point. All the timed results were acquired from a Linux workstation with a $2.8 \mathrm{GHz}$ Pentium IV processor and 2 GB memory.

We also have implemented the pruned Dijkstra's algorithm (also by Aleksandrov et al. [3]), which only uses an $\varepsilon$-spanner of each geodesic cone. Asymptotically, this algorithm is better than the cone-based Dijkstra's algorithm. However, it occurs to us that, for the $\varepsilon$ values we have chosen for our experiments, the pruned Dijkstra's algorithm is actually slower than the cone-based Dijkstra's algorithm. First of all, using the discretization $\mathcal{G}_{\varepsilon}$ that is guaranteed to contain an $\varepsilon$-good approximate optimal path, the pruned Dijkstra's algorithm can only guarantee to find an $5 \varepsilon$-good approximate optimal path, due to the extra error introduced by using the spanner. Therefore, to guarantee to find an $\varepsilon$-good approximate optimal path, the pruned Dijkstra's algorithm has to use the discretization $\mathcal{G}_{\varepsilon / 5}$, which contains many more Steiner points than $\mathcal{G}_{\varepsilon}$. Secondly, the pruned Dijkstra's algorithm needs to partition each geodesic cone into roughly $\frac{1}{\varepsilon}$ equal-sized angular ranges, so that one Steiner point can be picked inside each range. In our current implementation, this step will require $\frac{1}{\varepsilon}$ binary searches, which are very time-consuming. Before we come up with a more efficient implementation of the pruned Dijkstra's algorithm, we will not be able to correctly measure its performance.

\subsection{Experiments on TINs generated from DEM data}

One of the concerns in conducting these experiments is the choice of triangulations. We want to avoid randomly generated triangulations for two reasons. First, randomly generated data may affect the performances of various algorithms in an unexpected way. The second and main reason is that randomly generated triangulations may contain many "skewed" triangular regions (e.g., regions that are very skinny). Recall that the size of a discretization generated by either the discretization method of [3] or the one we presented in Section 6 is dependent on $\theta_{\min }$. If a certain experiment uses ten problem instances and for one problem instance $\theta_{\min }$ is very small, and much smaller than those of other problem instances, the experiment will take too much time to finish, and the result of this problem instance will dominate the result of the entire experiment.

For our experiments we chose triangulations converted from terrain maps in grid data format. More specifically, we used the DEM (Digital Elevation Model) file of Kaweah River basin. It is a $1424 \times 1163$ grid with $30 \mathrm{~m}$ between two neighboring grid points. From the DEM data we randomly took patches of sizes $8 \times 5,16 \times 10,32 \times 20,64 \times 40$, 


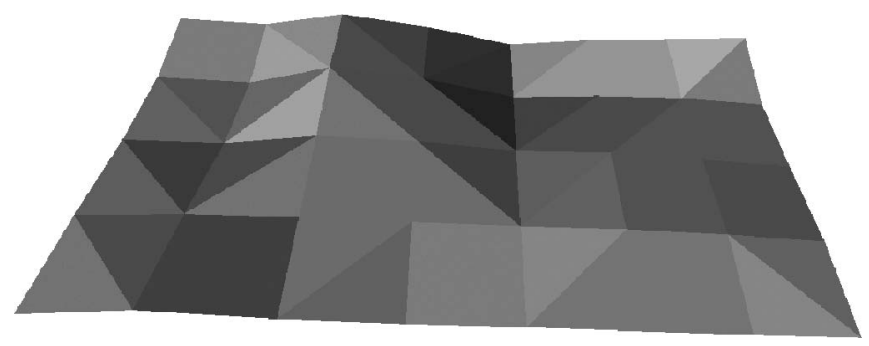

Fig. 12. A $8 \times 5$ TIN generated from DEM data.

and $128 \times 80$, respectively, (ten patches for each size) and converted them to TINs by connecting two grid points diagonally for each grid cell. Figure 12 shows one of the TINs we used.

Each triangular region of the resulting TINs will not be too skewed; its projection to the horizontal plane is an isosceles right triangle. We assign to each region $r$ a unit weight $w_{r}$ that is equal to $1+10 \tan \alpha_{r}$, where $\alpha_{r}$ is the angle between $r$ and the horizontal plane. We ran the three algorithms on each TIN with source and destination points chosen to be the vertices closest to the upper left and lower right corners, respectively. For each algorithm, we took the average of the running times of all experiments. We repeated the experiments with $\frac{1}{\varepsilon}=2,4,6,8$, and 10 . (For DEM size $128 \times 80$, we did not perform the experiments with $\frac{1}{\varepsilon}=8$ and 10.) We list the results in Table 3 .

From Table 3 , it is easy to see that, when $\frac{1}{\varepsilon}$ grows, the running time of the BUSHWHACK algorithm is growing much slower than those of both the pure Dijkstra's algorithm and the cone-based Dijkstra's algorithm. This observation is consistent with the asymptotic analysis, as the time complexity of the BUSHWHACK algorithm is less dependent on $\frac{1}{\varepsilon}$. We also list the average number of visited edges for each algorithm and each $\varepsilon$ value. It occurs to us that the number of visited edges is closely related to the running time.

We define the speedup ratio of the BUSHWHACK algorithm (with respect to the pure Dijkstra's algorithm) to be the ratio of computation time between the pure Dijkstra's algorithm and the BUSHWHACK algorithm. Table 3 shows that the speedup ratio of the BUSHWHACK algorithm drops slowly (but noticeably) when $n$ grows from 40 to 10240. For example, with $\frac{1}{\varepsilon}=4$, the speedup ratios are $1.6087,1.5553,1.4521,1.3732$, and 1.2686 for $n=40,160,640,2560$, and 10240, respectively. Recall that the time complexity of the pure Dijkstra's algorithm is $O\left(n m^{2}+n m \log n m\right)$, with $m$ being the number of Steiner points per edge. With a relatively large $m$ (even for $\left.\frac{1}{\varepsilon}=2\right)$, the first part $O\left(n m^{2}\right)$ dominates the second part $O(\mathrm{~nm} \log \mathrm{nm})$, and therefore the speedup ratio is proportional to $\frac{m}{\log n m}$ (roughly speaking). So for the values of $n$ we chose, the speedup ratio is relatively stable. If we choose a small $m$ (e.g., 20) and yet a large range for values of $n$ (e.g., up to $100,000)$, the speedup ratio would likely change more dramatically.

\subsection{Experiments on randomly generated TINs}

To demonstrate the impact of the geometric properties of TINs on the performance of the above algorithms, we also used twenty randomly generated TINs, ten with 40 vertices 
Table 3

Statistics of running time (in seconds) and number of visited edges (in thousands) on TINs generated from DEM data. Each entry of " $x / y$ " means that the average time for computing an $\varepsilon$-good approximate optimal path is $x$ seconds and the average number of visited graph edges is $y \times 1000$

\begin{tabular}{|c|c|c|c|c|}
\hline DEM size & $\frac{1}{\varepsilon}$ & BUSHWHACK & Pure Dijkstra & Cone-based Dijkstra \\
\hline \multirow{5}{*}{$8 \times 5$} & 2 & $0.48 / 140$ & $0.38 / 443$ & $0.60 / 360$ \\
\hline & 4 & $1.12 / 338$ & $1.80 / 2,288$ & $1.91 / 1,566$ \\
\hline & 6 & $1.85 / 566$ & $4.62 / 6,002$ & $3.85 / 3,340$ \\
\hline & 8 & $2.67 / 814$ & $9.07 / 11,874$ & $6.29 / 5,669$ \\
\hline & 10 & $3.57 / 1,077$ & $15.34 / 20,131$ & $7.92 / 8,463$ \\
\hline \multirow{5}{*}{$16 \times 10$} & 2 & $2.6 / 707$ & $1.9 / 2,216$ & $3.0 / 1,785$ \\
\hline & 4 & $6.1 / 1,712$ & $9.4 / 11,471$ & $9.5 / 7,905$ \\
\hline & 6 & $10.3 / 2,866$ & $24.3 / 30,094$ & $18.9 / 17,340$ \\
\hline & 8 & $15.0 / 4,122$ & $47.8 / 59,575$ & $31.1 / 29,903$ \\
\hline & 10 & $19.4 / 5,453$ & $80.2 / 101,026$ & $43.7 / 45,135$ \\
\hline \multirow{5}{*}{$32 \times 20$} & 2 & $13.5 / 3,201$ & $9.7 / 10,104$ & $12.6 / 8,025$ \\
\hline & 4 & $32.1 / 7,753$ & $46.6 / 52,357$ & $41.0 / 35,459$ \\
\hline & 6 & $54.6 / 12,974$ & $119.1 / 137,404$ & $82.2 / 78,343$ \\
\hline & 8 & $80.7 / 18,648$ & $231.8 / 271,988$ & $135.4 / 135,742$ \\
\hline & 10 & $98.0 / 24,669$ & $370.7 / 461,158$ & $203.0 / 206,351$ \\
\hline \multirow{5}{*}{$64 \times 40$} & 2 & $56.7 / 13,587$ & $41.8 / 42,715$ & $55.5 / 34,583$ \\
\hline & 4 & $138.5 / 32,895$ & $190.1 / 221,130$ & $180.5 / 154,902$ \\
\hline & 6 & $238.8 / 55,040$ & $479.5 / 580,293$ & $361.0 / 343,334$ \\
\hline & 8 & $347.0 / 79,107$ & $932.4 / 1,148,622$ & $595.3 / 595,967$ \\
\hline & 10 & $461.7 / 104,640$ & $1581.0 / 1,947,444$ & $901.4 / 906,556$ \\
\hline \multirow{3}{*}{$128 \times 80$} & 2 & $255.9 / 56,370$ & $181.1 / 179,639$ & $235.0 / 144,298$ \\
\hline & 4 & $647.8 / 136,342$ & $821.7 / 930,063$ & $764.9 / 647,184$ \\
\hline & 6 & $1132.4 / 227,965$ & $2050.2 / 2,440,243$ & $1530.8 / 1,439,499$ \\
\hline
\end{tabular}

and ten with 80 vertices. More specifically, for each TIN, we randomly selected 40 (80, respectively) vertices in the plane and then constructed a Delaunay triangulation with these vertices. For each triangular region of the resulting triangulation, we randomly assigned to it a weight between 1 and 10. Figure 13 shows one of the TINs generated. As one can see, some of the triangular regions are very long and skinny, meaning that many Steiner points need to be placed to guarantee a given error bound $\varepsilon$.

Again, for each TIN, we picked the source and destination points to be the vertices closest to the upper left and lower right corners, respectively. We ran each of the three algorithms on each TIN five times, with $\frac{1}{\varepsilon}=2,4,6,8$, and 10 , respectively. We list the results in Table 4.

Compared to the $8 \times 5$ DEM TINs mentioned in the previous subsection, the randomly generated TINs (with 40 vertices) have the same number of vertices and only slightly more boundary edges (108 vs. 95). However, for the same error bound $\varepsilon$, the number of Steiner points required for these TINs is roughly four times that for the DEM TINs. This is due to the fact that there exist some triangular regions with small angles, which boost the geometric parameters of the discretization. As a result, all the three algorithms performed worse on these TINs than on the DEM TINs. 


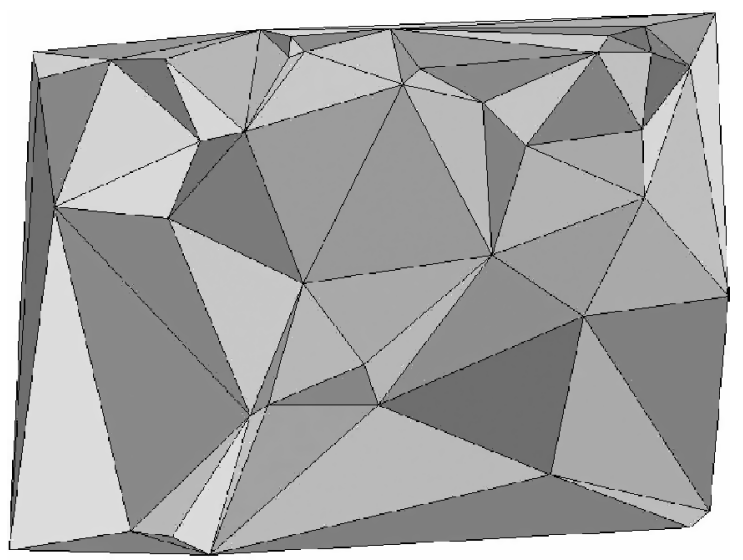

Fig. 13. A TIN generated by constructing a Delaunay triangulation of 40 randomly generated vertices.

Table 4

Statistics of running time (in seconds) and number of visited edges (in thousands) on randomly generated TINs. Each entry of " $x / y$ " means that the average time for computing an $\varepsilon$-good approximate optimal path is $x$ seconds and the average number of visited graph edges is $y \times 1000$

\begin{tabular}{ccccc}
\hline Number of vertices & $\frac{1}{\varepsilon}$ & BUSHWHACK & Pure Dijkstra & Cone-based Dijkstra \\
\hline \multirow{4}{*}{40} & 2 & $1.34 / 543$ & $14.85 / 16,148$ & $4.28 / 3,540$ \\
& 4 & $3.35 / 1,293$ & $78.37 / 85,512$ & $12.45 / 11,865$ \\
& 6 & $5.56 / 2,131$ & $203.87 / 223,508$ & $22.84 / 21,683$ \\
& 8 & $8.22 / 3,041$ & $399.52 / 439,442$ & $37.29 / 36,614$ \\
10 & $11.09 / 3,995$ & $682.22 / 740,040$ & $53.53 / 54,189$ \\
\hline \multirow{4}{*}{80} & 2 & $2.59 / 967$ & $11.27 / 11,921$ & $7.11 / 5,405$ \\
& 4 & $6.32 / 2,291$ & $57.22 / 61,284$ & $21.94 / 19,968$ \\
& 6 & $10.86 / 3,777$ & $146.54 / 158,344$ & $41.92 / 40,492$ \\
& 8 & $15.64 / 5,374$ & $288.76 / 309,282$ & $61.82 / 61,473$ \\
& 10 & $20.86 / 7,061$ & $490.83 / 518,641$ & $82.06 / 85,736$ \\
\hline
\end{tabular}

In particular, the pure Dijkstra's algorithm suffered the most. For example, with $\frac{1}{\varepsilon}=8$, the speedup ratio of the BUSHWHACK algorithm (with respect to the pure Dijkstra's algorithm) is just 3.4033 for the DEM TINs, but grows to 48.6237 for the randomly generated TINs. This result is also consistent with the asymptotic analysis, as the time complexity of the pure Dijkstra's algorithm is more sensitive to the number of Steiner points. What makes it worse is that in a randomly generated TIN one or a few of the (long and skinny) triangular regions will get a significant portion of all Steiner points, making the computation by the pure Dijkstra's algorithm even more time-consuming. Recall that the $O\left(\mathrm{~nm}^{2}\right)$ part of the time complexity of the pure Dijkstra's algorithm assumes that each boundary edge contains roughly the same number of Steiner points. The actual upper bound should be the total number of face-crossing segments connecting discrete points on the same region boundary. If the total number of Steiner points is still $O(\mathrm{~nm})$, and yet each of the two long boundary edges of one very skewed triangular region gets $\frac{n m}{4}$ Steiner points, then the 
number of face-crossing segments in this region is already $\frac{n^{2} m^{2}}{16}$, which could already be more than the $O\left(\mathrm{~nm}^{2}\right)$ bound.

Another evidence of the high sensitivity of the pure Dijkstra's algorithm on the geometric parameters is that on average it took even more time to finish the TINs with 40 vertices than those with 80 vertices. This is because some of TINs with 40 vertices have "worse" triangular regions (with very small angles) than those with 80 vertices do.

\subsection{Observations}

From the experimental results we learned that, although for the discretization we used the number of Steiner points inserted on each boundary edge is $O\left(\frac{1}{\varepsilon} \log \frac{1}{\varepsilon}\right)$, the hidden constant factor, which is determined by some geometric parameters, is very large. For example, for $\varepsilon=\frac{1}{8}$, the number of Steiner points on each boundary edge is on the order of a few hundreds. The reason is that, for discretization based on edge-subdivision, the error of an approximate optimal path is bounded "locally." The approximate optimal path and the corresponding optimal path are divided into many subpaths, and the weighted length of each subpath of the approximate optimal path has to be no more than $(1+\varepsilon)$ times of that of the corresponding subpath of the optimal path. All the subpaths of the approximate optimal path usually cannot contribute an $\varepsilon$-relative error simultaneously (and some of the subpaths may even be less costly than their counterparts in the optimal path), the overall relative error of the approximate optimal path is far less than $\varepsilon$.

That being said, we believe that this discretization scheme based on edge subdivision is still very useful in practice. It is very easy to implement, and can accommodate different cost metrics. For example, Lanthier et al. [19] showed that with minor modifications a discretization can be constructed for the shortest anisotropic path problem, while Reif and Sun [20] presented a similar discretization for the optimal path planning problem in regions with flows.

\section{Conclusion}

In this paper we present a new approximation algorithm to solve the weighted shortest path problem. Compared to some of the previous work, our algorithm provides a more effective way of finding optimal paths in the discretized space (resulted from either uniform or non-uniform discretization). We also provide an improved discretization method that removes the dependency of the size of the discretized space (and hence the complexity of any approximation algorithm that uses the discretization method) on the unit weight ratio.

\section{References}

[1] M. Lanthier, A. Maheshwari, J.-R. Sack, Approximating weighted shortest paths on polyhedral surfaces, in: Proceedings of the 13th Annual ACM Symposium on Computational Geometry, 1997, pp. 274-283.

[2] L. Aleksandrov, M. Lanthier, A. Maheshwari, J.-R. Sack, An $\varepsilon$-approximation algorithm for weighted shortest paths on polyhedral surfaces, in: Proceedings of the 6th Scandinavian Workshop on Algorithm Theory, in: Lecture Notes in Comput. Sci., vol. 1432, 1998, pp. 11-22.

[3] L. Aleksandrov, A. Maheshwari, J.-R. Sack, Approximation algorithms for geometric shortest path problems, in: Proceedings of the 32nd Annual ACM Symposium on Theory of Computing, 2000, pp. 286-295. 
[4] J.H. Reif, Z. Sun, An efficient approximation algorithm for weighted region shortest path problem, in: Proceedings of the 4th Workshop on Algorithmic Foundations of Robotics, 2000, pp. 191-203.

[5] J.S.B. Mitchell, Geometric shortest paths and network optimization, in: J.-R. Sack, J. Urrutia (Eds.), Handbook of Computational Geometry, Elsevier/North-Holland, Amsterdam, 2000, pp. 633-701.

[6] J.S.B. Mitchell, C.H. Papadimitriou, The weighted region problem: Finding shortest paths through a weighted planar subdivision, J. ACM 38 (1) (1991) 18-73.

[7] C. Mata, J.S.B. Mitchell, A new algorithm for computing shortest paths in weighted planar subdivisions, in: Proceedings of the 13th Annual ACM Symposium on Computational Geometry, 1997, pp. 264-273.

[8] T.R. Smith, G. Peng, P. Gahinet, Asynchronous, iterative, and parallel procedures for solving the weightedregion least cost path problem, Geographical Analysis 21 (2) (1989) 147-166.

[9] R. Alexander, Construction of optimal-path maps for homogeneous-cost-region path-planning problems, PhD thesis, Computer Science, US Naval Postgraduate School, Monterey, CA, 1989.

[10] R. Alexander, N. Rowe, Geometrical principles for path planning by optimal-path-map construction for linear and polygonal homogeneous-region terrain, Technical Report, Computer Science, US Naval Postgraduate School, Monterey, CA, 1989.

[11] R. Alexander, N. Rowe, Path planning by optimal-path-map construction for homogeneous-cost twodimensional regions, in: Proceedings of the 1990 IEEE International Conference on Robotics and Automation, 1990, pp. 1924-1929.

[12] M. Kindl, M. Shing, N. Rowe, A stochastic approach to the weighted-region problem, II: Performance enhancement techniques and experimental results, Technical Report, Computer Science, US Naval Postgraduate School, Monterey, CA, 1991.

[13] N. Papadakis, A. Perakis, Minimal time vessel routing in a time-dependent environment, Transportation Science 23 (4) (1989) 266-276.

[14] N. Papadakis, A. Perakis, Deterministic minimal time vessel routing, Oper. Res. 38 (3) (1990) 426-438.

[15] N.C. Rowe, Roads, rivers, and obstacles: optimal two-dimensional route planning around linear features for a mobile agent, Internat. J. Robotics Res. 9 (6) (1990) 67-74.

[16] N.C. Rowe, R.F. Richbourg, An efficient Snell's Law method for optimal-path planning across multiple two-dimensional, irregular, homogeneous-cost regions, Internat. J. Robotics Res. 9 (6) (1990) 48-66.

[17] Z. Sun, J.H. Reif, BUSHWHACK: An approximation algorithm for minimal paths through pseudoEuclidean spaces, in: Proceedings of the 12th Annual International Symposium on Algorithms and Computation, in: Lecture Notes in Comput. Sci., vol. 2223, 2001, pp. 160-171.

[18] L. Aleksandrov, A. Maheshwari, J.-R. Sack, An improved approximation algorithm for computing geometric shortest paths, in: Proceedings of the 14th International Symposium on Fundamentals of Computation Theory, in: Lecture Notes in Comput. Sci., vol. 2751, 2003, pp. 246-257.

[19] M. Lanthier, A. Maheshwari, J.-R. Sack, Shortest anisotropic paths on terrains, in: Proceedings of the 26th International Colloquium on Automata, Languages and Programming, in: Lecture Notes in Comput. Sci., vol. 1644, 1999, pp. 524-533.

[20] J.H. Reif, Z. Sun, Movement planning in the presence of flows, Algorithmica 39 (2) (2004) 127-153. 